\title{
The Importance of the Debye Bosons (Sound Waves) for the Lattice Dynamics of Solids
}

\author{
U. Köbler \\ Research Center Jülich, Institute PGI, 52425 Jülich, Germany \\ E-mail: u.koebler@fz-juelich.de
}

Received 22 Nov 2019, Revised 3 Mar 2020, Accepted 23 Mar 2020

\begin{abstract}
For non-magnetic solids the two experimental signatures of a non-negligible and decisive interaction between the Debye bosons (sound waves) and the (acoustic) phonons are discussed: 1.) for large thermal energies the dispersion of the mass-less Debye bosons is a weaker than linear function of wave vector, and 2.) for many cubic materials the dispersion of the acoustic phonons along $\left[\begin{array}{lll}\zeta & 0 & 0\end{array}\right]$ direction follows a perfect sine function of wave vector, which is known to be the dispersion of the linear atomic chain. Only the absolute phonon energies are due to the inter-atomic interactions. It is argued that the sine-function originates in a relatively weak Debye boson-phonon interaction. For a strong Debye boson-phonon interaction, the dispersion of the acoustic phonons assumes initially over a large q-range the linear dispersion of the Debye bosons, followed by an analytical crossover to the sine-function. As a consequence of the boson-controlled wave-vector dependence of the phonons, the temperature dependence of the heat capacity of the phonon system is also determined by the Debye bosons, and exhibits universal power functions of absolute temperature. Quantitative analyses of the dispersion relations of the mass-less Debye bosons (sound waves) of cubic materials along $\left[\begin{array}{lll}\zeta & 0 & 0\end{array}\right]$ direction show that the dispersion is a linear function of wave vector only for low energies. When all phonon modes are excited, that is, for thermal energies of larger than corresponds to the Debye temperature $\left(\Theta_{\mathrm{D}}\right)$, the dispersion of the Debye bosons follows a power function of wave-vector $\sim \mathrm{q}^{\mathrm{x}}$. For the exponent $\mathrm{x}$ the rational values of $x=0,1 / 4,1 / 3,1 / 2,2 / 3$ and $3 / 4$ could firmly be established experimentally. The discrete values of $x$ show that there are distinct modes of interaction with the phonons only. Quantitative analyses show that the temperature dependence of the heat capacity can be described accurately over a large temperature range by the expression $\mathrm{c}_{\mathrm{p}}=\mathrm{c}_{0^{-}}$ $\mathrm{B} \cdot \mathrm{T}^{-\varepsilon}$. The constants $\mathrm{c}_{0}$ and $\mathrm{B}$ are material specific and define the absolute value of the heat capacity. However, for the exponent $\varepsilon$ the same rational value can be observed for materials with different chemical compositions and lattice structures. The finite temperature range of the $c_{p}=c_{0}-B \cdot T^{-\varepsilon}$ function and the rational exponents $\varepsilon$ are the typical characteristics of a boson determined universal behavior. This universality must, however, be considered as a nonintrinsic dynamic property of the atomistic phonon system, arising from the Debye boson-phonon interaction. Safely identified values for $\varepsilon$ are $\varepsilon=1,5 / 4$ and $4 / 3$. The discrete modes of the boson-phonon interaction are essential for the different universality classes of the heat capacity, i.e. for the different exponents $\varepsilon$. The fit values for $c_{0}$ are generally larger than the theoretical Dulong-Petit value. Universal exponents are identified also in the temperature dependence of the coefficient of the linear thermal expansion, $\alpha(T)$. Since the universal power functions in the $\alpha(T)$ dependence are functions of absolute temperature and hold for the same thermal energies (temperatures) as the $\sim \mathrm{q}^{\mathrm{x}}$ functions in the dispersion of the Debye bosons, it can be concluded that the Debye bosons determine also the temperature dependence of $\alpha(T)$. Our results show that the Debye bosons dominate the dynamics of the atomic lattice of the nonmagnetic solids for all temperatures. The atomistic models restricting on the inter-atomic interactions therefore are neither sufficient to explain the phonon dispersion relations nor the detailed temperature dependence of the heat capacity.
\end{abstract}

Keywords: Lattice dynamics; boson fields; universality. 


\section{Introduction}

The temperature dependence of the heat capacity of all non-magnetic solids is qualitatively very similar, quite independent of the chemical composition and of the lattice structure. Only the absolute values of the heat capacity are material specific and are determined by the microscopic interatomic interaction strengths and by the total number of the atomic degrees of freedom. The total number of degrees of freedom defines the saturation value of the heat capacity at the Dulong-Petit (D-P) limit, given by $c(D-P)=3 n N_{L} k_{B}$ where $n$ is the number of atoms per formula unit, $\mathrm{N}_{\mathrm{L}}$ the number of atoms per mole and $\mathrm{k}_{\mathrm{B}}$ the Boltzmann constant. The interatomic interaction strengths define the temperature at which the D-P limit is reached. This occurs approximately at the Debye temperature $\Theta_{\mathrm{D}}$.

A temperature dependence that is independent of the chemical composition and of the lattice structure is called universal. Observation of universality strongly suggests that the temperature dependence of the heat capacity (not its absolute value!) is controlled by a boson field (see Figures 18-20 below). The only known boson type that can be made responsible for the observed universal temperature dependence of the heat capacity are the Debye bosons, better known as sound waves. Universality for temperatures for which the absolute values of the heat capacity are material specific and are determined by the inter-atomic interactions, is surprising [1]. Only the low-temperature heat capacity of all solids is well-known to be universal [2]. For all solids, the observed low-temperature heat capacity is the heat capacity of the Debye boson field, and follows the famous Debye $\mathrm{T}^{3}$ function over a finite temperature range, commonly up to $10 \ldots 30$ $\mathrm{K}$ (see Figure 1). In terms of the Renormalization Group (RG) methods [3], in this temperature range the heat capacity of the material specific lattice vibrations is not relevant and is completely suppressed. In other words, there is no thermal energy in the system of the lattice vibrations (phonons). It is a quite general, and experimentally well-established issue of the RG-methods, that in the vicinity of critical temperatures the dynamics is that of a boson field. The microscopic inter-atomic interactions play no role. In fact, $\mathrm{T}=0$ is the critical temperature of the Debye boson field, completely equivalent to a finite ordering temperature. The $\mathrm{T}^{3}$ function could be called a critical power function, and is a prominent example of universality (Figure 1 ). Note that by symmetry arguments, phonons and bosons cannot become relevant at the same time.
No contribution of the lattice vibrations to the observed heat capacity can be proven by showing that the pre-factor of the $\mathrm{T}^{3}$ function agrees with the pre-factor calculated from the known sound velocities [4]. Possible contributions of the lattice vibrations would increase the heat capacity over the Debye value (see Figure 2). For the following it is important to note that the low-temperature $\mathrm{T}^{3}$ function is the intrinsic behavior of the Debye boson field. Observation of the same $\mathrm{T}^{3}$ function in solids with different lattice symmetries is because the Debye boson field is not ordered and, so to say, isotropic. Typical for disordered boson fields is the absence of domains.

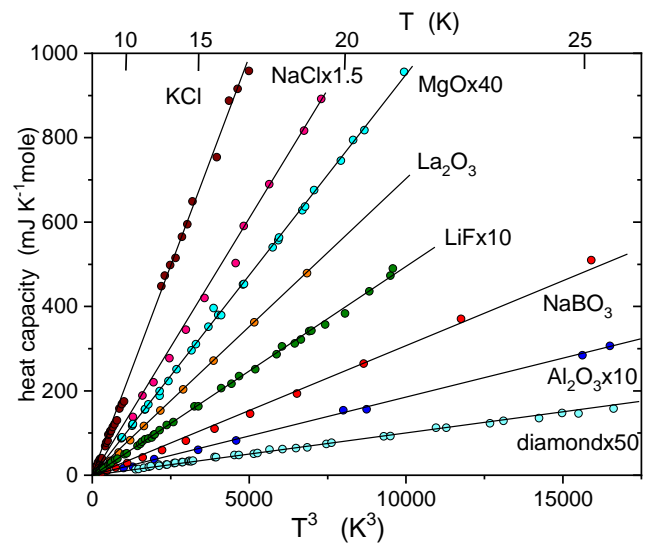

Figure 1. Low-temperature heat capacities of various insulating compounds with different compositions and lattice structures as a function of the absolute temperature to a power of three [8]. For the mechanically hard materials the lowtemperature heat capacity is low (the Debye temperature is high) and has been multiplied by the given factors. The material-independent universal $T^{3}$ function is the intrinsic heat capacity of the Debye boson field. Heat capacity contributions of the lattice are not relevant, in the sense of the RG-methods.

In the metals an additional heat capacity term, linear-in-temperature, due to the electronic system superimposes on the $\mathrm{T}^{3}$ term [5]. Since the linear-in$\mathrm{T}$ term occurs for all metals and holds approximately over the same temperature range as the $\mathrm{T}^{3}$ function of the Debye boson field, it is also due to bosons [6]. These bosons are the excitations of the spatially continuous electronic (metallic) medium. Quite analogous to the Debye bosons that exist in addition to the phonons, the bosons of the electronic continuum (the EC-bosons) exist in addition to the better-known electronic band states [6]. In fact, in solids we have to distinguish between the bosons of the elastic, magnetic and electronic degrees of 
freedom. Superposition of the $\mathrm{T}^{3}$ and of the T-term in the low-temperature heat capacity of all metals [5] is possible only because the Debye bosons and the EC-bosons do nearly not interact. Due to the very weak interaction with the elastic degrees of freedom, the EC-bosons have a very large mean free path [7]. The fact that thermal conductivity of all metals starts with the linear-in-T dependence of the heat capacity of the EC-bosons proves that thermal conductivity of the metals is exclusively due to the EC-bosons. The mean free path of the EC-bosons therefore must be larger than the usual linear dimension of the samples $[6,7]$. Note that thermal conductivity by phonons is generally negligible [7]. Observation of a linear-in$\mathrm{T}$ heat capacity for all metals, independent of the lattice structure shows that the EC-boson field is not ordered. As for the Debye boson field, the critical temperature of the EC-boson field is $\mathrm{T}=0$; the critical heat capacity (the linear-in $\mathrm{T}$ term) is a function of absolute temperature. Only for temperatures of larger than $10 \ldots 30 \mathrm{~K}$ the crossover to the atomistic band-states occurs and the dispersion of the ECbosons is no longer thermally populated. Thermal conductivity of the metals then decreases dramatically [7]. The nearly temperatureindependent low thermal conductivity for $\mathrm{T}>10 \ldots 30$ $\mathrm{K}$ is due to the electronic band states.

Figure 1 visualizes for a selection of insulators the composition- and lattice structure independence of the universal $\mathrm{T}^{3}$ function of the low temperature heat capacity [8]. A power function of temperature with rational exponent that holds over a finite temperature range is the typical signature of a boson field. In fact, universality is the thermodynamic behavior of a boson field [6]. Universality results from the latticestructure independent, ballistic propagation mode of the bosons. This makes the dispersion relation of the bosons a single power function of wave-vector for all energies. For the mass-less Debye boson, the dispersion is a linear function of wave-vector along all crystallographic directions, at least for low thermal energies (compare Figures 8-15 below).

The Debye $\mathrm{T}^{3}$ function holds rather precisely up to a crossover at which the pre-factor of the $\mathrm{T}^{3}$ function increases suddenly. Figure 2 visualizes this analytical crossover event using the heat capacity of $\mathrm{MgO}$ as an example [9]. We have called this type of crossover amplitude crossover (AC) [6]. For the rather hard material $\mathrm{MgO}$ the amplitude crossover is at a fairly high temperature of $\mathrm{T}_{\mathrm{AC}}=40 \mathrm{~K}$. Due to the increased pre-factor of the $\mathrm{T}^{3}$ function for temperatures of $\mathrm{T}>\mathrm{T}_{\mathrm{AC}}$ the heat capacity becomes larger, over an intermediate temperature range, compared to the Debye heat capacity [10]. The amplitude crossover therefore provides clear evidence for the existence of energy degrees of freedom in addition to the Debye bosons. These energy degrees of freedom are, of course, the acoustic phonons. The increased pre-factor of the $\mathrm{T}^{3}$ function for $\mathrm{T}>\mathrm{T}_{\mathrm{AC}}$ is a nice experimental example to illustrate the principle of the RG-methods to distinguish between relevant and non-relevant energy degrees of freedom [3]. For $\mathrm{T}<\mathrm{T}_{\mathrm{AC}}$ the Debye bosons are the relevant excitations. In this temperature range the observed heat capacity is the intrinsic heat capacity of the Debye boson field and follows a pure $\mathrm{T}^{3}$ function. The heat capacity of the lattice vibrations is negligible (if the pre-factor of the $\mathrm{T}^{3}$ function agrees with the pre-factor calculated from the sound velocities [4]). With increasing thermal energy, or temperature, the dispersion energy of the Debye bosons becomes larger than the dispersion energy of the acoustic phonons. This is because the dispersion of the mass-less Debye bosons continues as a nearly linear function of wave vector, while the dispersion of the acoustic phonons is curved and saturates towards the zone boundary (see Figures 9-13 below). The finite atomic nearneighbor interactions set an upper energy limit to the phonons. Since the inter-atomic interactions are of no importance for the Debye bosons, their (nearly) linear dispersion continues unlimited. Only the melting temperature sets an upper energy limit to the Debye bosons. Note that the melting temperature commonly is much higher than conforms to the largest phonon energy, given approximately by $\sim \mathrm{k}_{\mathrm{B}} \cdot \Theta_{\mathrm{D}}$ (see Figures 8-15 below). It is intuitively clear that at high temperatures the system with the lowest excitation energy will host the thermal energy. At elevated temperatures this is the phonon system. This qualitative argument becomes quantitatively stringent due to the symmetry selection principle of relevance. According to the RG methods [3], the thermal energy can be only either in the system with discrete translation symmetry (phonons) or in the system with continuous translation symmetry (Debye boson field). Since there is a finite interaction between the two systems, the two symmetries exclude each other. Relevance, therefore, has the dramatic consequence that all available states of the boson field and of the atomistic excitations (phonons) cannot simultaneously be occupied thermally according to the Boltzmann factor. Detailed balance holds for the energy level system of the relevant excitation systems only. The energy states of the non-relevant system are thermally not populated. The alternation of the thermal energy from one to the other excitation 
system is a crossover that gives rise to a non-steady behavior in the temperature dependence of the heat capacity.

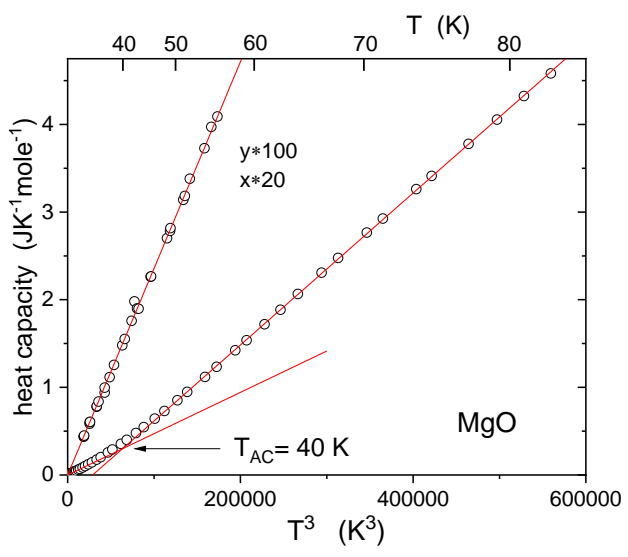

Figure 2. Amplitude crossover at $T_{A C}=40 \mathrm{~K}$ in the $T^{3}$ dependence of the heat capacity of $\mathrm{MgO}$ [9]. Only for $T>T_{A C}$ the heat capacity of the non-relevant phonons is sufficiently large to induce a crossover to a larger pre-factor of Debye's universal $T^{3}$ function. Low-temperature data are plotted a second time with the coordinate values multiplied by the given factors.

The transfer of the thermal energy from the Debye boson field to the system of the lattice vibrations proceeds by two crossover events [6]. We will designate the two crossover temperatures by $\mathrm{T}_{\mathrm{AC}}$ and $\mathrm{T}^{*}$. For $\mathrm{T}>\mathrm{T}_{\mathrm{AC}}$, the Debye boson field remains, at first, the relevant excitation system over some finite temperature range. This means, the $\mathrm{T}^{3}$ function persists. However, the effect of the non-negligible but non-relevant acoustic phonons is to increase the pre-factor (amplitude) of the universal $\mathrm{T}^{3}$ function. The phonons now are no longer completely suppressed. The amplitude crossover illustrates the principle of the RG methods that finite but nonrelevant energy degrees of freedom enter the prefactor of the universal power function of temperature of the relevant system but they do not change the universal exponent that is determined by the relevant system [3]. The nature and the intrinsic heat capacity of the non-relevant system therefore become not apparent. For the following it is important to note that this is a typical example that the heat capacity of the material specific lattice vibrations is dominated by the Debye bosons. The heat capacity contributions of the Debye bosons and of the phonons do not superimpose. This is because the two systems with different translational symmetry interact. The amplitude crossover is a threshold induced, discrete reaction on a sufficiently strong continuous variation of non-relevant energy contributions as a function of temperature. This proves the stability of the universality class of the boson field and its dynamic dominance. Only when the non-relevant energy contribution has become as strong as the relevant energy contribution it can become relevant and can change or lift the universality class. In this way, the type of the dynamic symmetry is always clearly defined, also when two excitation systems with different (translational) symmetry interfere. Typical for the non-asymptotic $\mathrm{T}^{3}$ function is an absolute (negative) constant (see Figure 2).

When the phonons are the relevant excitation system, the $\mathrm{T}^{3}$ function does no longer hold. This happens at a second crossover event, that is at $\mathrm{T}^{*}=92$ $\mathrm{K}$ in the case of $\mathrm{MgO}$, and therefore is outside the temperature window of Figure 2. At $\mathrm{T}^{*}$ the transfer of the thermal energy to the phonon system gets completed (see Figures 3 and 4) [6]. At this crossover, the heat capacity falls below the asymptotic Debye $\mathrm{T}^{3}$ function and, eventually, saturates at the D-P limit. The two crossover events at $\mathrm{T}_{\mathrm{AC}}=40 \mathrm{~K}$ and at $\mathrm{T}^{*}=92 \mathrm{~K}$ (in the case of $\mathrm{MgO}$ ) with an increasing and a decreasing heat capacity with respect to the asymptotic $\mathrm{T}^{3}$ Debye heat capacity, respectively, give rise to a rather sharp minimum in the conventional descriptions of the experimental heat capacity data by a temperature dependent Debye temperature [11]. Note that a large heat capacity means a low Debye temperature. A continuous temperature dependence of the fitted Debye temperature means an infinite number of fit parameters.

Commonly it is assumed that the heat capacity shows a material specific, atomistic dynamics and, therefore, exhibits no universal power functions of temperature when the atomistic phonons determine the absolute value of the heat capacity [1]. This argument refers to the temperature interval between $\mathrm{T}=\mathrm{T}^{*}$ and that temperature at which the heat capacity reaches the D-P limit which occurs approximately at $\mathrm{T}=\Theta_{\mathrm{D}}$ (Figures 3 and 4 ). It is the aim of this communication to show on account of quantitative analyses of available experimental heat capacity data $[8,14,15]$ that this is not correct. Obviously, the Debye bosons interact at all thermal energies (temperatures) with the phonon system and determine the wave-vector dependence of the phonons (see Figures 5-7 below) and, therefore, the heat capacity of the phonon system. The interactions between the Debye bosons and the phonons do obviously not depend on whether the dispersion relation of the Debye bosons is thermally populated or not, i.e. whether the Debye bosons are relevant or 
not. Note that for temperatures of $\mathrm{T}>\mathrm{T}^{*}$ the dispersion of the Debye boson is no longer thermally populated (the $\mathrm{T}^{3}$ function does no longer hold) (Figures 3 and 4). In the temperature range $\mathrm{T}>\mathrm{T}^{*}$, the Debye bosons are, so to say, virtual states. Owing to the Debye boson-phonon interaction that is active for all temperatures, the heat capacity of the phonon system receives a non-intrinsic universal temperature dependence from the Debye bosons. Essential for the distinct universality classes of the heat capacity of the phonon system is that there are only a few discrete modes of the Debye bosonphonon interaction. The different modes of interaction make the dispersion of the Debye bosons a non-linear function of wave vector $\left(\sim q^{x}\right)$ with a rational exponent $\mathrm{x}$. As we will see, depending on the type of interaction, the discrete values of the exponent $x$ are in the wide range of $0<x<3 / 4$. This enables a rather clear distinction between the different exponent values. The universality classes induced by the Debye bosons in the temperature dependence of the heat capacity of the phonon system therefore depend essentially on the type of interaction between Debye bosons and phonons. It is observed that the exponents $\varepsilon$ in the temperature dependence of the heat capacity of the phonon system according to $\mathrm{c}_{\mathrm{p}}=\mathrm{c}_{0}-\mathrm{B} \cdot \mathrm{T}^{-\varepsilon}$ are rational numbers (see Figures 18-20 below). Note that the parameters $\mathrm{c}_{0}$ and $\mathrm{B}$ are atomistic, i.e. material specific and define the absolute value of the heat capacity. The parameter $c_{0}$ scales approximately with the D-P limiting value, i.e. with the total number of atomic degrees of freedom, while the parameter $\mathrm{B}$ is determined by the inter-atomic interaction strengths and scales approximately with $\Theta_{\mathrm{D}}$ (see Figure 18 below). Since all observed values of the exponent $\varepsilon$ are in the narrow window of $1<\varepsilon<4 / 3$, they are more difficult to separate from each other experimentally than the corresponding exponents $\mathrm{x}$ in the dispersion of the Debye bosons. The decisive characteristic of a boson defined universal heat capacity of the phonon system is that a single power function of absolute temperature with rational exponent holds over a large temperature range (see Figures 18-20 below). It seems to be a general phenomenon that the dynamics of solids is determined by bosons [6]. The length scale of the boson-determined dynamics is given by the mean free path (or coherence length) of the bosons. Phonons (or magnons) are local excitations on the short length scale of the lattice parameter and provide no unambiguous evidence of a coherent long-range order (see below). On the length scale of the inter-atomic distance there are no universal features.
Indications of rather weak Debye boson-phonon interactions are already noticeable for the temperature range of Debye's asymptotic $\mathrm{T}^{3}$ function. The $\mathrm{T}^{3}$ function holds exactly only for a vanishing Debye boson-phonon interaction. Note that in the temperature range of the $\mathrm{T}^{3}$ function the heat capacity of the phonons is suppressed. In fact, the $\mathrm{T}^{3}$ function results from a pure field theory assuming that the field bosons are perfectly free particles and have linear dispersion [2]. This means that the velocity of the Debye bosons is a temperature independent constant. In reality, this holds strictly for $\mathrm{T} \rightarrow 0$ only [13]. Debye's theory is very similar to Planck's treatment of the electromagnetic radiation field in vacuum. Note that the Planck boson field is universal, i.e. independent of the material of the walls of the cavity. Its energy density is $\sim \mathrm{T}^{4}$ (Stefan-Boltzmann law) for all temperatures.

As is well-known, the assumption of perfectly free bosons is rarely realized in solids. For practically all solids it is observed that the sound velocities (elastic constants) decrease moderately as a function of an increasing temperature [12]. This is because the effective interaction of the Debye bosons with the phonons becomes stronger with an increasing thermal excitation of the phonons. This interaction provides damping to the Debye bosons and decreases their velocity as a function of an increasing temperature [13]. Additionally, damping of the Debye bosons depends somewhat on the purity and crystalline perfection of the considered sample, and therefore is not perfectly reproducible [12]. Quite generally, due to interactions with the atomic background the dispersion of the Debye bosons becomes a weaker than linear function of wavevector [13]. Debye's $\mathrm{T}^{3}$ function then holds ideally for vanishing interactions only, that is asymptotically for $\mathrm{T} \rightarrow 0$. In the range $\mathrm{T}<\mathrm{T}_{\mathrm{AC}}$, the deviations from the perfect $\mathrm{T}^{3}$ function are rather small and not resolved in Figure 1.

With increasing temperature more and more phonons get excited. Eventually, on approaching the Debye temperature $\Theta_{\mathrm{D}}$, nearly all phonons are excited. The Debye boson-phonon interaction then has reached its full value. This qualitative change is associated with an analytical crossover in the dispersion relation of the Debye bosons. Approximately at $E \sim \mathrm{k}_{\mathrm{B}} \cdot \Theta_{\mathrm{D}}$ the dispersion of the Debye bosons changes from a weakly non-linear to a strongly non-linear wave-vector dependence (Figures 8-15). The strongly curved dispersion section can well be described by the expression $E(q)=-A+B \cdot q^{x}$. Since this $q-$ function holds for the 
large energy range between the Debye temperature, $\Theta_{\mathrm{D}}$, and the melting temperature, $\mathrm{T}_{\mathrm{m}}$, rather accurate fits for the exponent $x$ are possible. It turns out that the fit values for the exponent $\mathrm{x}$ are rational numbers to a very good approximation. This is the basis for the rational values of the exponent $\varepsilon$ in the temperature dependence of the heat capacity of the phonon system $\left(c_{p}=c_{0}-B \cdot T^{-\varepsilon}\right)$.

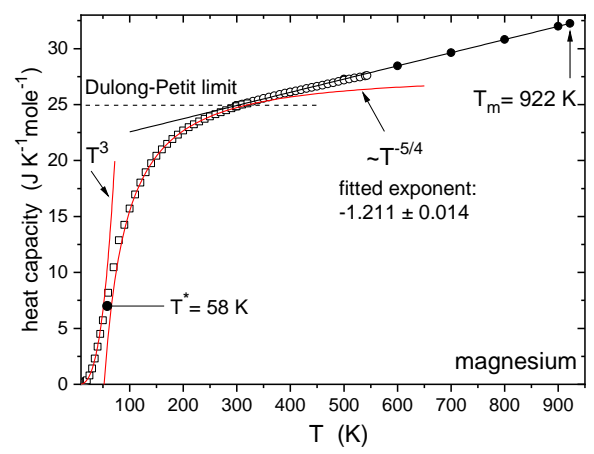

Figure 3. At $T^{*}=58 \quad K$ the heat capacity of magnesium exhibits an analytical crossover from Debye's $T^{3}$ function to a power function of type $c_{p}=c_{0}-B \cdot T^{\varepsilon}$. Near to the Dulong-Petit limit a further crossover occurs (change from red curve to black straight line). The heat capacity values of larger than the D-P limit must be attributed to another type of boson field (the MP-bosons) that determines the critical dynamics at the melting point (see text) [16]. For many materials the heat capacity above the D-P limit is a linear function of temperature up to melting point [14-16].

In summary, in the temperature dependence of the heat capacity of the insulating and non-magnetic solids three crossover events can be identified. At each crossover an analytical change of the temperature function occurs. Consequently, in contrast to the misleading concept of Debye [2], there is no closed expression possible that would describe the heat capacity for all temperatures. At a first crossover, at $\mathrm{T}_{\mathrm{AC}}$, the pre-factor of the $\mathrm{T}^{3}$ function increases suddenly (Figure 2). At a second rather broad crossover, at $\mathrm{T}^{*}$, the $\mathrm{T}^{3}$ function ceases and a change to a power function of temperature of the form $\mathrm{c}_{\mathrm{p}}=\mathrm{c}_{0}-\mathrm{B} \cdot \mathrm{T}^{-\varepsilon}$ occurs (Figures 3 and 4 ). Note that in Figures 3 and 4, the amplitude crossover events of magnesium $\left(\mathrm{T}_{\mathrm{AC}}=18.5 \mathrm{~K}\right)$ and of aluminum $\left(\mathrm{T}_{\mathrm{AC}}=22 \mathrm{~K}\right)$ are not resolved. For the metals, the visualization of the amplitude crossover as in Figure 2 requires subtraction of the electronic heat capacity $\left(c_{\mathrm{el}}=\gamma \cdot \mathrm{T}\right)$ from the measured heat capacity $[5,6]$. Since the bosons of the electronic continuum, the EC-bosons, interact neither with the Debye bosons nor with the phonons there seems to be no amplitude crossover in the electronic heat capacity such that the $c_{e l}=\gamma \cdot T$ function holds up to $T^{*}$. This allows for a convenient subtraction of $c_{e l}$ from the observed heat capacity of the metals [6]. Note that at $\mathrm{T}^{*}$, the $\mathrm{T}^{3}$ function of the Debye bosons and the $\mathrm{T}$-function of the EC-bosons cease. For magnesium we find $\gamma=1.455 \mathrm{mJK}^{-2} \mathrm{~mole}^{-1}$ and for aluminum $\gamma=1.38 \mathrm{mJK}^{-}$ ${ }^{2} \mathrm{~mole}^{-1}$.

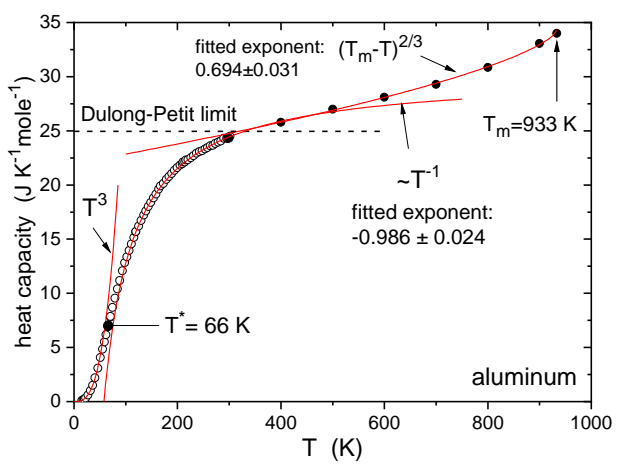

Figure 4. Heat capacity of aluminum up to melting point at $T_{m}=933 \mathrm{~K}$. At $T^{*}=66 \mathrm{~K}$ a crossover from $T^{3}$ function to a power function of type $c_{p}=c_{0}-B \cdot T^{1}$ occurs [14,15]. At the Dulong-Petit limit of 24.95 $J^{-1}$ mole ${ }^{-1}$, a further crossover to a power function of type $c_{p}=A-B \cdot\left(T_{m}-T\right)^{2 / 3}$ occurs. The constant $A$ gives the heat capacity at the melting temperature $T_{m}$. Note that $c_{0}$ is larger than the D-P limit.

We should mention that the fitted values for $\mathrm{c}_{0}$ are generally larger by approximately $\sim 10 \%$ compared to the theoretical D-P limit (see Figures 3 and 4). A third crossover occurs near to that temperature at which the heat capacity exceeds the D-P limit. This is approximately at $\Theta_{D}$. For magnesium, $\Theta_{D}=403 \mathrm{~K}$, for aluminum $\Theta_{D}=433 \mathrm{~K}$. Since heat capacity values of larger than the D-P limit are beyond atomistic concepts, it follows that additional energy degrees of freedom must be responsible for these heat capacity values. The large temperature range of the linear temperature dependence in Figure 3 and of the power function $c_{p}=A-B \cdot\left(T_{m}-T\right)^{2 / 3}$ in Figure 4 provides evidence that these power functions are also due to a boson field with $\mathrm{T}_{\mathrm{m}}$ as critical temperature. Further examples of the power functions of the argument $\sim\left(\mathrm{T}_{\mathrm{m}}-\mathrm{T}\right)$ can be found in [16]. Note that as for the range of the low-temperature $\mathrm{T}^{3}$ function, the heat capacity values of larger than the D-P limit are exclusively due to a boson field. We will call these bosons that determine the heat capacity in the vicinity of the melting point, MP-bosons. The hypothetical MP-bosons are, however, completely unexplored. We must assume that the MP-boson 
field orders at $\mathrm{T}_{\mathrm{m}}$ and therefore appears to be responsible for the development of a long-range and coherent atomic order below $\mathrm{T}_{\mathrm{m}}$. This argument assumes that the MP-bosons are also generated by stimulated emission. Typical for ordered boson fields are domains. It is suggestive to consider the mosaic blocks, occurring in practically all solids, as the domains of the ordered MP-boson field. We should note that in axial crystals the domain structures are not very stable and can depend on the strain in the sample. As a consequence, the domain structure can easily be changed by weak external perturbations such as pressure. Note that the longrange and coherent atomic order reveals clearly only from the very narrow x-ray scattering lines observed in the crystal structure analyses. Phonon (or magnon) dispersions measured by inelastic neutron scattering are local excitations and give no unambiguous evidence of a long-range atomic (magnetic) order. Moreover, the MP-bosons warrant the cohesion of the solid for temperatures of larger than corresponds to the inter-atomic interaction strengths, as they can be estimated from the largest observed phonon energies, that correspond approximately to $k_{B} \cdot \Theta_{D}$. Note that $T_{m}$ is generally larger than $\Theta_{D}$. In other words, the MP-bosons have binding function and prevent the decomposition of the solid. This is different for the Goldstone bosons in magnetism. The Goldstone bosons destabilize the magnetic order and shift the magnetic ordering temperature to a lower value than corresponds to the near-neighbor exchange interactions. In ferromagnets, the critical temperature $\mathrm{T}_{\mathrm{C}}$ is generally lower than the CurieWeiss temperature $\Theta$. Possibly, all order-disorder phase transitions are driven, by boson fields $[18,19]$. Typical for boson driven phase transitions is that the width of the critical range is finite and that the critical exponents are rational numbers. This seems to apply also to phase transitions of first order such as the melting transition $[16,17]$.

In the intermediate temperature range between $T^{*}$ and the D-P limit, the lattice vibrations determine the absolute values of the heat capacity. Nevertheless, in this temperature interval, the detailed temperature dependence of the heat capacity is determined by the Debye boson field, and is given by a power function of absolute temperature $\left(c_{\mathrm{p}}=\mathrm{c}_{0}-\mathrm{B} \cdot \mathrm{T}^{-\varepsilon}\right)$. If in this temperature range the MP-bosons would determine the temperature dependence of the heat capacity, power functions of $\left(\mathrm{T}_{\mathrm{m}}-\mathrm{T}\right)$ should hold. Note that the Debye boson field is not ordered, its critical temperature is $\mathrm{T}=0$. The heat capacity of the Debye boson field is a function of absolute temperature! This also supports the conclusion that the function of absolute temperature for the phonon heat capacity according to $\mathrm{c}_{\mathrm{p}}=\mathrm{c}_{0}-\mathrm{B} \cdot \mathrm{T}^{-\varepsilon}$ is determined by the interaction with the Debye bosons.

That bosons control the dynamics of atomistic systems is well-know from magnetism [6]. The absolute values of the spontaneous magnetization are given by the atomic magnetic moments and are material specific but the thermal decrease of the spontaneous magnetization is determined not by the interactions between the spins but by the heat capacity of the Goldstone boson field and exhibits universality [6]. Independence the boson-controlled universal dynamics of the near-neighbor exchange interactions reveals clearly from the fact that in the whole ordered range the dynamics is independent of the spin structure. The width of the critical range at the two critical temperatures $\mathrm{T}=0$ and the magnetic ordering temperature, $\mathrm{T}=\mathrm{T}_{\mathrm{c}}$ is sufficiently large such that the two associated critical power functions overlap [6]. The intersection point of the two power functions is another example of a crossover event (analytical change). For most order-disorder phase transition, such as the liquid-gas transition, the relevant bosons are, however, not yet explored [18,19].

The exponent $\varepsilon$ in the temperature function $\mathrm{c}_{\mathrm{p}}=\mathrm{c}_{0^{-}}$ $\mathrm{B} \cdot \mathrm{T}^{-\varepsilon}$ of the heat capacity of the phonons is universal. Since this universality is not an intrinsic property of the phonon system but arises from the Debye bosonphonon interaction, the exponent $\varepsilon$ depends on the type of this interaction. As a consequence of the different types of boson-phonon interaction, different values for $\varepsilon$ result. Since the heat capacity is an integral quantity, it is affected by all interactions between Debye bosons and phonons along all crystallographic directions and for all polarizations. Here we restrict on the evaluation of these interactions for cubic materials along [ $\left.\begin{array}{lll}\zeta & 0 & 0\end{array}\right]$ direction. This is accomplished by fitting $\sim \mathrm{q}^{\mathrm{x}}$ power functions to the high-energy part of the dispersion relations of the Debye bosons (see Figures 8-15 below). The corresponding analyses along the other crystallographic directions are more complex and less conclusive. Clearly identified values for the exponent $\mathrm{x}$ are $\mathrm{x}=0,1 / 4,1 / 3,1 / 2,2 / 3$ and 3/4. From our analyses of the heat capacity according to the function $\mathrm{c}_{\mathrm{p}}=\mathrm{c}_{0}-\mathrm{B} \cdot \mathrm{T}^{-\varepsilon}$, exponent values of $\varepsilon=1,5 / 4$ and $4 / 3$ could be established firmly (see Figures 16-18) but further exponents between $\varepsilon=1$ and $\varepsilon=5 / 4$ seem to exist in addition. It is, however, not possible to correlate the exponents $\mathrm{x}$, evaluated from the dispersions of the Debye bosons along only the $[\zeta, 0$, 0 ] direction with the integral exponent $\varepsilon$. Note that 
the exponents $x$ commonly are different for the two polarizations of the Debye bosons.

\section{Dispersion of the Acoustic Phonons}

The following analyses of the dispersion relations of the acoustic phonons of cubic materials along $[\zeta 0$ $0]$ direction provide strong evidence that the wavevector dependence of the phonon dispersions is determined by the Debye bosons. Since atoms have a mass, excitation of lattice vibrations requires irradiation of massive neutrons. Figure 5 shows for the cubic Rare Gas solids and for solid ${ }^{4} \mathrm{He}$ the dispersions of the phonons with transverse polarization measured along cube edge using inelastic neutron scattering [20-24]. All shown solid gases have the fcc structure. As can be seen, for all materials the dispersion is given perfectly by a sine function of wave vector (solid curves through the experimental points). As is well-known, the sine function is the dispersion of the linear atomic chain. It is unlikely that the linear chain dispersion is the intrinsic behavior of three-dimensional solids. From the perfect one-dimensional dispersion, we have to conclude that the generation process of the Debye bosons is dominated, as for the Goldstone bosons in the magnetic solids, by stimulated emission $[6,27,28,41]$. The sine function evidently holds independent of the type of the solid gas and of the details of the inter-atomic interactions. Only the prefactor of the sine function is material specific.

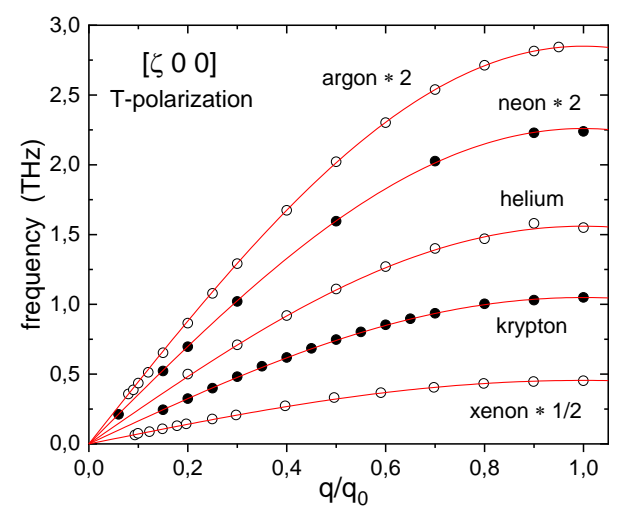

Figure 5. Dispersion of the phonons with transverse polarization of some solid gases, all with cubic fcc structure, measured along cube edge using inelastic neutron scattering [20-24]. The curves through the experimental points are sine functions of wavevector. These data have been taken at $10 \mathrm{~K}\left({ }^{36} \mathrm{Ar}\right.$, Xe, $\mathrm{Kr})$, at $6.5 \mathrm{~K}(\mathrm{Ne})$, and at $15.5 \mathrm{~K}\left({ }^{4} \mathrm{He}\right)$. For better clarity the dispersion energies of $\mathrm{Ne}$ and $\mathrm{Xe}$ are multiplied by the given factors.
Figure 6 shows similar results but for conventional solids all with cubic lattice symmetry [25]. For all selected materials the dispersion of the acoustic phonons with transverse polarization is given by a sine function of wave-vector (solid curves through the experimental points). Note that the materials of Figure 6 have different cubic lattice structures: copper has fcc structure, $\mathrm{LiF}$ and $\mathrm{TiC}$ have the $\mathrm{NaCl}$ structure with 4 molecules per unit cell, $\mathrm{CsCl}$ has cubic structure with only one molecule per unit cell [26]. As a conclusion, the sine function of wave-vector is universal and must, hence, be determined by a boson field. The only bosons that one could imagine are the Debye bosons

A phonon dispersion that is manipulated by a boson field has the severe consequence that the interatomic force constants cannot be evaluated conclusively from the phonon dispersion curves [1]. We should mention, that a similar phenomenon is observed for the magnetic excitations (magnons) $[6,27,41]$. For many magnets with one magnetic species only, the dispersion of the magnons is given by a sine-function of wave vector for antiferromagnets but by a sine-function squared for ferromagnets [41]. These functions are known to apply to the linear spin chains. Observation of onedimensional magnon dispersion relations in threedimensional solids can be explained by the fact that the magnon propagation is restricted to the individual magnetic domain. Since the Goldstone bosons (magnetic dipole radiation) are generated by stimulated emission [28], the boson field within each domain is perfectly one-dimensional [27]. Owing to interactions with the spin system, the onedimensional boson field stabilizes the perfect collinear spin alignment within each magnetic domain and leads to the one-dimensional wave vector dependence of the magnons, quite independent of the local anisotropy of the exchange interactions and of the lattice structure. In other words, the one-dimensional Goldstone boson field furnishes the spin system with a particular axial anisotropy that is not known in the atomistic spin wave theory. In magnets with a pure spin moment, the magnon gap provides a measure of the stability of the one-dimensional spin order. We must assume that the functionality of the Debye bosons for the dispersion of the phonons is similar to the functionality of the Goldstone bosons for the dispersion of the magnons, although the Debye boson field is not ordered. Note that magnon dispersions as for the linear spin chain are observed also in the paramagnetic phase where the Goldstone boson field is also not ordered [27,28]. For 
disordered boson fields the observed dimensionality seems to hold on the short atomistic length scale only, as it is sampled specifically by inelastic neutron scattering [41].

Since the initial slopes of the sine functions in Figure 6 agree quantitatively with the quoted sound velocities, measured at the same temperature as the phonons [12], it can be concluded that the Debye bosons determine the initial slopes of the phonon dispersion. Note that the sound velocities are measured with a completely independent experimental method compared to the phonon dispersions. The results of the two experimental methods are combined in Figure 6. In other words, due to a finite Debye boson-phonon interaction the dispersion relations of the two excitation systems attract each other. It is a quite common phenomenon that the dispersion relations of excitations with different (translation) symmetries cannot cross but can attract each other only, if there is a finite interaction between them. Crossing is allowed only for a vanishing interaction. We can assume that not only the slope of the initially linear dispersion of the acoustic phonons is determined by the Debye bosons, but that the whole wave vector dependence (the sine-function) is determined by the Debye bosons. From the fact that the dispersion of the phonons is as for the linear atomic chain we have to conclude that the Debye boson field is onedimensional, along the main crystallographic symmetry directions. As for the Goldstone bosons (magnetic dipole radiation) in magnetism [6,28], the spontaneous generation of the Debye bosons seems to be dominated by stimulated emission. Spontaneous creation and absorption of Debye bosons by individual atoms are, however, completely unexplored processes.

Since the Debye boson field is not ordered (the critical temperature is $\mathrm{T}=0$ ), the long-range coherent atomic order, evidenced by $\mathrm{x}$-ray scattering methods, cannot be stabilized by the Debye bosons but must be stabilized by the ordered MP-boson field. Note that in the critical paramagnetic range above the magnetic ordering temperature, the Goldstone boson field is also not ordered. In spite of no long-range magnetic order, the Goldstone bosons are the relevant excitations to determine the universality class (the critical exponent) of the two-spin correlation length and of the paramagnetic susceptibility. These quantities can be sampled using the microscopic method of neutron scattering. The observed one-dimensional structures of the disordered boson fields, possibly, hold on the short atomic length scale only, and seem to be a consequence of the generation process of the bosons by stimulated emission. Note that magnons and phonons are the typical excitations on the short length scale of the lattice parameter. Magnons and phonons provide no definite evidence of a longrange and coherent order. This can be concluded from the fact that magnons can be observed far above the magnetic ordering temperature $[33,41]$.

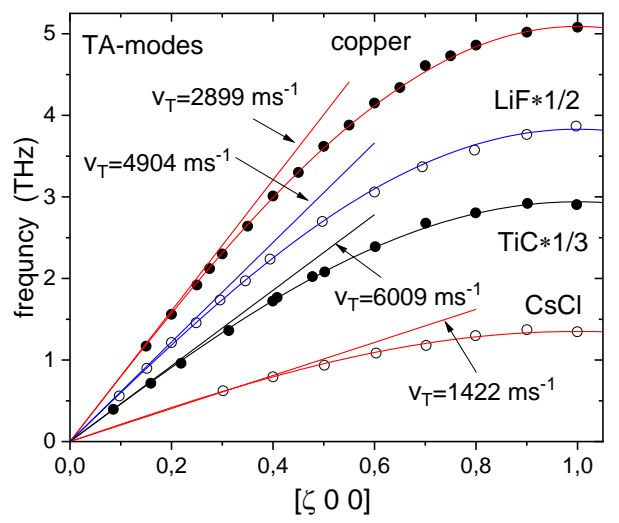

Figure 6. For the selected cubic materials, the dispersion of the acoustic phonons with transverse polarization measured along the cube edge is given by a sine-function of wave vector (solid curves), quite independent of the chemical composition and of the details of the lattice structure viz. inter-atomic interactions [29-32]. The initial slopes agree quantitatively with the sound velocities (straight lines) measured at the same temperature as the phonon dispersions [12]. Only for $\mathrm{CsCl}$ the phonon dispersion is measured at $78 \mathrm{~K}$, for the other materials at room temperature.

The behavior displayed Figures 5 and 6 is not generally observed. A dispersion given by a pure sine function of wave vector for all q-values seems to apply to materials with a rather weak Debye boson-phonon interaction only. For a stronger Debye boson-phonon interaction the dispersion of the acoustic phonons gets stronger attracted by the linear dispersion of the Debye bosons. This means, the phonon dispersion initially assumes over a finite wave vector range the linear dispersion of the Debye bosons. The slope of this linear dispersion section agrees with the measured sound velocities (Figure 7) [12]. The strong modification of the phonon dispersion by the Debye bosons shows the primacy of the Debye bosons. Since either the behavior of Figure 6 or Figure 7 is observed, it can be concluded that the Debye boson-phonon interaction is quantized. 


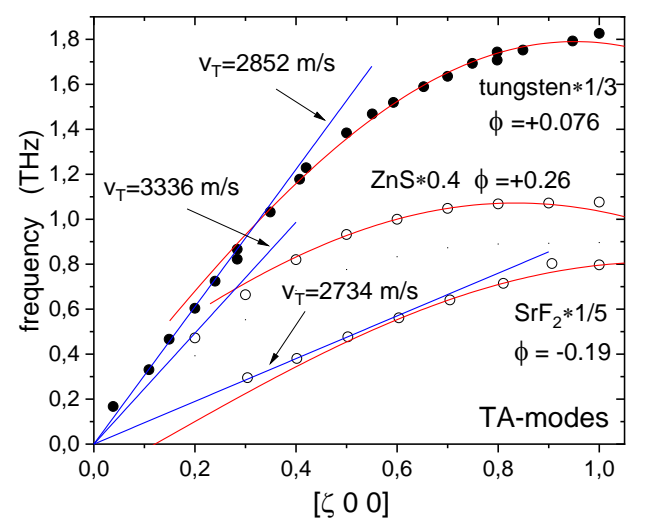

Figure 7. For a strong Debye boson-phonon interaction the dispersion relation of the acoustic phonons is determined over a finite wave vector range by the linear dispersion of the Debye bosons [25,34-36]. At the end of the linear section an analytical crossover to a sine function occurs [27]. It turns out to be necessary to add a phase shift ( $\phi)$ in the argument of the sine function. The given sound velocities refer to ambient temperature at which the phonon dispersions are taken.

A linear phonon dispersion over a finite q-range implies that an analytical crossover must occur at the end of the linear dispersion section. At this crossover the dispersion changes to a sine function of wave vector. However, in order to obtain good agreement with the experimental data it turns out to be necessary to add a phenomenological phase shift $(\phi)$ in the argument of the sine-function [6,27]. The phase shift can be positive or negative and seems to be another measure of the Debye boson-phonon interaction strength. This clearly proves that the Debye boson-phonon interaction affects the dispersion of the acoustic phonons for all q-values. Due to the phase shift the zone boundary is no longer an absolutely sharp limit for the phonons. This is a typical consequence of the Debye boson-phonon interaction. Note that the Debye bosons propagate independent of the lattice structure. The zone boundary is of no significance for the Debye bosons. As will be shown by Figures 8-15 in compound materials the dispersion of the Debye bosons extends much beyond the zone boundary.

In the case of a very strong Debye boson-phonon interaction the analytical crossover from the linear dispersion to the sine function gets shifted to a large q-value. The q-range of the sine function then is small. Fitting the dispersion data by a sine function, including a phase shift in the argument, then gets very difficult or even impossible.

\section{Dispersion of the Debye Bosons}

Since the Debye bosons (sound waves) have no mass they cannot be observed using scattering of massive neutrons. Instead, sound waves can be excited using transmitters attached to the surface of the sample. On the other hand, sound injection by a transmitter can generally not excite lattice vibrations. As we have seen in the preceding Chapter, Debye boson-phonon interactions modify the dispersion of the acoustic phonons. According to the principle of reciprocity it can therefore be expected that the dispersion of the Debye bosons will correspondingly be modified by the Debye bosonphonon interaction.

The dispersion of the mass-less Debye bosons cannot be measured directly. However, as we have explained in [13], the dispersion of the Debye bosons can be constructed from the known temperature dependence of the sound velocities that are simply related to the elastic constants [12]. Using this method, it is assumed that the sound velocity measured at a temperature $\mathrm{T}$ gives the slope of the dispersion curve at an excitation energy of $\mathrm{k}_{\mathrm{B}} \cdot \mathrm{T}$. For all solids it is observed that the sound velocities (elastic constants) decrease somewhat as a function of an increasing temperature [12]. This effect can be attributed to interactions of the Debye bosons with the phonon system. With increasing thermal excitation of the phonons, the effective Debye boson-phonon interaction increases. This interaction provides an enhanced damping to the Debye bosons with increasing temperature and decreases their velocity. As a consequence, the dispersion of the Debye bosons assumes a weaker than linear wavevector dependence.

On the other hand, it is well-known that the elastic properties of solids depend somewhat on the crystalline perfection and on the impurity content of the sample, and therefore are not perfectly reproducible. Fortunately, for high-quality crystals, the intrinsic damping mechanism owing to interactions of the Debye bosons with the lattice vibrations dominates. The effect of the lattice imperfections is to decrease the absolute values of the elastic constants by a small nearly constant amount [12]. The dominant contribution to the temperature dependence of the sound velocity seems to be due to the intrinsic mechanism of the Debye boson-phonon interactions.

As a first example of the dispersion of the Debye bosons, calculated from the temperature dependence of the sound velocities, Figure 8 displays data for cubic $\mathrm{KCl}[12]$. For energies of larger than $\sim \mathrm{k}_{\mathrm{B}} \cdot \Theta_{\mathrm{D}}$ 
the dispersion of the Debye bosons becomes strongly non-linear. This is characteristic for a strong Debye boson-phonon interaction. Evidently, for a strong Debye boson-phonon interaction all phonons must be excited. This is the case for thermal energies of $\mathrm{E}>\mathrm{k}_{\mathrm{B}} \cdot \Theta_{\mathrm{D}}$. In fact, approximately at $\mathrm{E} / \mathrm{k}_{\mathrm{B}} \sim \Theta_{\mathrm{D}}$ the heat capacity exhibits also a crossover (Figures 3 and 4). At this crossover the MP-bosons become the relevant excitations for larger temperatures. On the other hand, for thermal energies of lower than $\sim \mathrm{k}_{\mathrm{B}} \cdot \Theta_{\mathrm{D}}$ the dispersion of the Debye bosons is much less curved. We have to identify the change from a nearly linear but weakly curved dispersion for $\mathrm{E}<\mathrm{k}_{\mathrm{B}} \cdot \Theta_{\mathrm{D}}$ to a strongly curved dispersion for $\mathrm{E}>\mathrm{k}_{\mathrm{B}} \cdot \Theta_{\mathrm{D}}$ as an analytical crossover event. It turns out that for energies of $E>k_{B} \cdot \Theta_{D}$ the dispersion of the Debye bosons can well be fitted by $q / q_{0}=-A+B \cdot q^{x}$. Note that the $q / q_{0}=-A+B \cdot q^{x}$ function is for an energy range where the MP-bosons are the relevant excitations. Nevertheless, there are strong indications that the Debye bosons interact not with the MP-bosons but with the phonons. One argument for this is the analytical form of the dispersion relation according to $\mathrm{q} / \mathrm{q}_{0}=-\mathrm{A}+\mathrm{B} \cdot \mathrm{q}^{\mathrm{x}}$ that provides evidence that the Debye bosons interact with the phonons (see below). As we will show on discussing Figure 17, the MPbosons seem not to couple to the elastic degrees of freedom. No interaction of the MP-bosons with the elastic degrees of freedom (Debye bosons) might explain the fact that the melting transition of all solids is first order [15]. Ordering of the MP-boson field at the melting temperature resembles the threshold for the onset of stimulated emission of a LASER.

Note that for cubic crystals the velocity of the sound waves with longitudinal polarization, propagating along the cube edge (the $\left[\begin{array}{lll}1 & 0 & 0\end{array}\right]$ direction) is given by $\mathrm{v}_{\mathrm{L}}=\left(\mathrm{c}_{11} / \rho\right)^{1 / 2}$ and for sound waves with transverse polarization by $\mathrm{v}_{\mathrm{T}}=\left(\mathrm{c}_{44} / \rho\right)^{1 / 2}$ with $\rho$ as mass density. For no interaction of the Debye bosons with the phonons the elastic constants would be temperature independent and the dispersion of the Debye bosons would be a linear function of wave vector for all q-values (for all thermal energies). A constant sound velocity is realized for $\mathrm{T} \rightarrow 0$ (or $\mathrm{q} \rightarrow 0$ ) only. In Figure 8 , the slope of this initially linear wave vector dependence is given by the straight lines for $\mathrm{q} \rightarrow 0$. The associated sound velocities are given.

The power function $\mathrm{q} / \mathrm{q}_{0}=-\mathrm{A}+\mathrm{B} \cdot \mathrm{q}^{\mathrm{x}}$ holds over the rather large energy range, starting from $E \sim k_{B} \cdot \Theta_{D}$ up to a thermal energy that corresponds to the melting temperature $\left(\sim \mathrm{k}_{\mathrm{B}} \cdot \mathrm{T}_{\mathrm{m}}\right)$. The absolute constant indicates that the function $\mathrm{q} / \mathrm{q}_{0}=-\mathrm{A}+\mathrm{B} \cdot \mathrm{q}^{\mathrm{x}}$ is not the asymptotic behavior for $\mathrm{q}_{\mathrm{q}} \mathrm{q}_{0} \rightarrow 0$. The fitted experimental values for the exponent $\mathrm{x}$ are to a very good approximation rational numbers of lower than unity. For $\mathrm{KCl}$, the fitted values for $\mathrm{x}$ are consistent with the assumed rational numbers of $x=1 / 2$ and $3 / 4$. Since we attribute the curvature of the dispersion of the Debye bosons to the interactions with the phonons it follows from the discrete values of the exponents $\mathrm{x}$ that there are distinct modes of interaction only. The lower the exponent $\mathrm{x}$ is, compared to unity, the stronger is the Debye bosonphonon interaction.

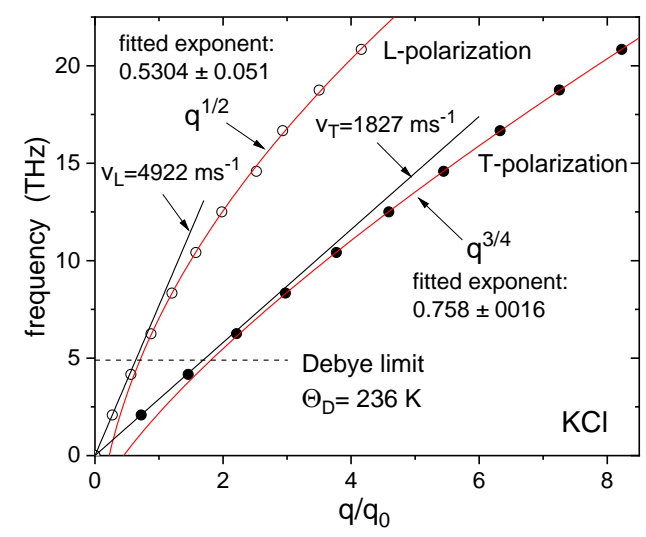

Figure 8. Dispersion of the mass-less Debye bosons with longitudinal (circles) and transverse polarization (filled circles) along the cube edge of $\mathrm{KCl}$ as constructed from the temperature dependence of the sound velocities, calculated from the elastic constants $c_{11}(T)$ and $c_{44}(T)$, respectively [12,13]. After a nearly linear dispersion at small $q$ values an analytical crossover to $a \sim q^{x}$ function (including a negative constant) with rational exponent $x$ is identified (for explanation see text). The given sound velocities are calculated for $c_{11}(T \rightarrow 0)$ and $c_{44}(T \rightarrow 0)$.

There are several very remarkable features with the results displayed by Figure 8. Very surprising is that the dispersion of the Debye bosons extends much beyond the zone boundary, $\mathrm{q} / \mathrm{q}_{0}=1$. In principle, the shortest possible wave-length of the bosons, and therefore the largest value of $q / q_{0}$ is given by the diameter of the sources of the bosons. We must assume that the sources of the Debye bosons are the individual atoms, possibly inner atomic shells. As a consequence, for compound materials, the largest wave vector of the Debye bosons can be larger than the zone boundary that is given by the lattice parameter, $\mathrm{q}_{0}=2 \pi / \mathrm{a}_{0}$. On the other hand, for $\mathrm{KCl}$, the dispersion of the Debye bosons 
with transverse polarization, extends up to $\mathrm{q} / \mathrm{q}_{0} \sim 8$. Since the lattice parameter of $\mathrm{KCl}$ is $\mathrm{a}_{0}=6.293 \AA$ [26], this means that the diameter of the sources of the Debye bosons with transverse polarization is $\sim 0.8 \AA$ which is considerably smaller than the diameter of the $\mathrm{K}^{+}$ion $(\sim 2.66 \AA)$ or the diameter of the $\mathrm{Cl}^{-}$ion $(\sim 3.62 \AA)$. Note that for the $\mathrm{NaCl}$ structure of $\mathrm{KCl}$ the lattice parameter is given by the sum of the diameters of the $\mathrm{K}^{+}$ion and of the $\mathrm{Cl}^{-}$ion.

Another very revealing detail in Figure 8 (and in all following Figures) is that the dispersion energy of the Debye bosons continues monotonically with increasing wave vector up to an energy that conforms to the melting temperature, $\mathrm{T}_{\mathrm{m}}$. For $\mathrm{KCl}$, $\mathrm{k}_{\mathrm{B}} \cdot \mathrm{T}_{\mathrm{m}}$ corresponds to $\sim 22 \mathrm{THz}[8,15]$. In particular, no anomaly can be seen at the Debye cut-off energy of $k_{B} \cdot \Theta_{D}$ with $\Theta_{D}=236 \mathrm{~K}$ for $\mathrm{KCl}$ [4]. The monotonous behavior of the dispersion of the Debye bosons at the cut-off energy of $\mathrm{k}_{\mathrm{B}} \cdot \Theta_{\mathrm{D}}$ corresponds to the monotonous temperature dependence of the elastic constants $c_{11}(\mathrm{~T})$ and $\mathrm{c}_{44}(\mathrm{~T})$ that, commonly, are measured up to the melting temperature $\mathrm{T}_{\mathrm{m}}$ [12]. As can be seen in Figures 8-15, quite generally, the fit of the $q / q_{0}=-A+B \cdot q^{x}$ power functions holds up to melting temperature.

Absence of any anomaly at Debye's cut-off energy proves the unphysical nature of this cutting procedure. The artificial termination of the dispersion of the Debye bosons at $\mathrm{k}_{\mathrm{B}} \cdot \Theta_{\mathrm{D}}$ has the nonsensical physical consequence that the solid would no longer have elastic properties for temperatures of $\mathrm{T}>\Theta_{\mathrm{D}}$. As a consequence, all solids would be ideal soundproofing materials for temperatures of larger than the Debye temperature. This contradicts the empirical facts. As is well-known, all solids have elastic properties up to the melting temperature and beyond as well. This means, excitation of sound waves is possible for all temperatures. The intention of Debye with his cutting procedure was to stop the exploding $\mathrm{T}^{3}$ dependence of the heat capacity of the Debye boson field. As we now know, the $\mathrm{T}^{3}$ function ceases at that temperature at which the thermal energy changes from the Debye boson field into the system of the lattice vibrations. As we have explained in connection with Figures 3 and 4, this crossover is at $\mathrm{T}^{*}$. At the time of Debye, crossover events were unknown, mainly because sufficient experimental data were not available to provide the necessary information. According to the symmetry selection principle of relevance, established by the RG methods [3], the thermal energy can be only in one or the other of the two excitation systems. Note that bosons and phonons are distinguished by different translational symmetries. In other words, the principle of relevance ascertains that different symmetries exclude each other.

It was the conceptual shortcoming of Debye's theory not to strictly distinguish between the continuous translation symmetry of the bosons (sound waves) and the discrete translation symmetry of the phonons. At the time of Debye, these two symmetries were not yet recognized as the generators of different particles with specific excitation spectra. In his field theory, Debye has cut the assumed perfect linear dispersion of the sound waves in such a way that the heat capacity of the boson field saturates at the atomistic Dulong-Petit limit. To this end it was necessary to limit the infinite number of states of the boson field to the absolute number of states of the atomic system. The incorporation of atomic parameters, such as the number of atomic states, into a field theory is, however, strictly forbidden. The inconsistency of this procedure lies in the fact that atoms do not occur in a field theory. This is a consequence of the fact that the field bosons propagate ballistic, that is, independent of the atomic structure. For the bosons the solid is a continuum. Note that saturation of the heat capacity at the D-P limit holds automatically when all thermal energy has changed to the system of the lattice vibrations. The heat capacity of the Debye boson field then tends to zero.

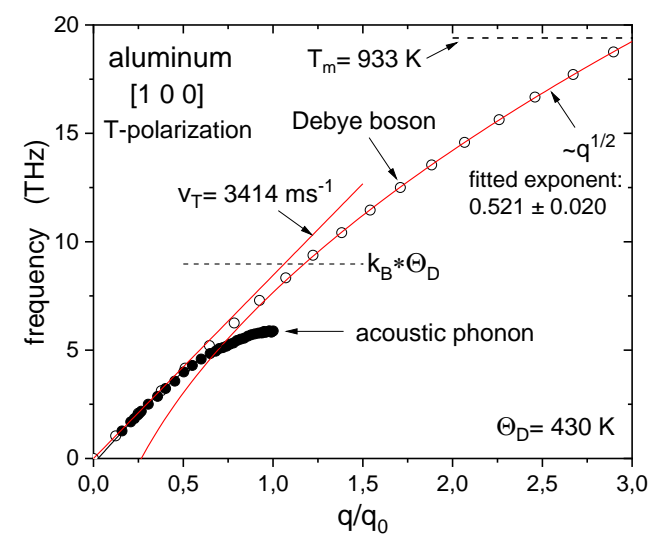

Figure 9. Dispersion of the Debye bosons (circles) [12,13] and of the acoustic phonons (filled circles) [37] of aluminum, both with transverse polarization, measured along the edge of the cubic fcc unit cell as a function of the reduced wave-vector. For $q / q_{0}>1$ the dispersion of the Debye bosons is excellently described by a wave vector dependence as $\sim q^{1 / 2}$. Note that the dispersion of the Debye bosons is continuous at Debye's cut-off energy of $k_{B} \cdot \Theta_{D} \sim 8.9$ $\mathrm{THz}$. 
In the following Figures 9-15 further examples of the evaluation of the exponents $\mathrm{x}$ are presented. Figure 12 shows that in one case $(\mathrm{LiF})$ the particular exponent $\mathrm{x}=0$ has been observed. This means a logarithmic behavior.

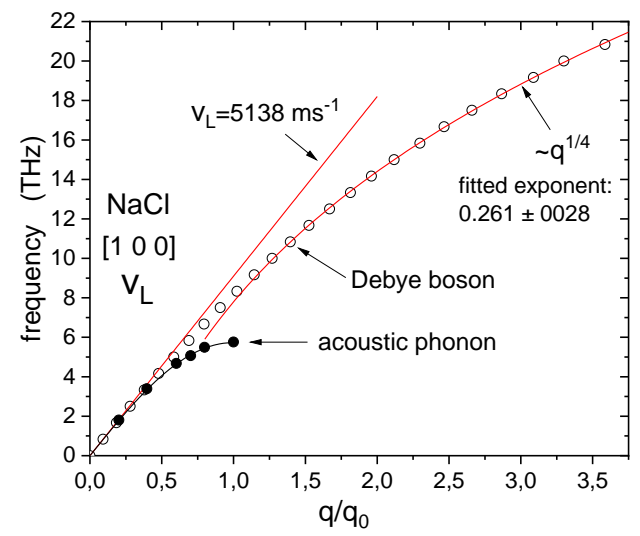

Figure 10. Dispersion of the acoustic phonons (filled circles), measured at $T=80 \mathrm{~K}$ [38] and dispersion of the Debye bosons (circles) [12], both for longitudinal polarization, measured along the edge of the cubic unit cell of $\mathrm{NaCl}$. The velocity of the Debye bosons at $T=80 \mathrm{~K}$, calculated from the elastic constant $c_{11}(T)$, is indicated as straight line [12]. Above the analytical crossover at $q / q_{0} \sim 1$, the dispersion data of the Debye bosons are excellently described by the rational exponent of $x=1 / 4$.

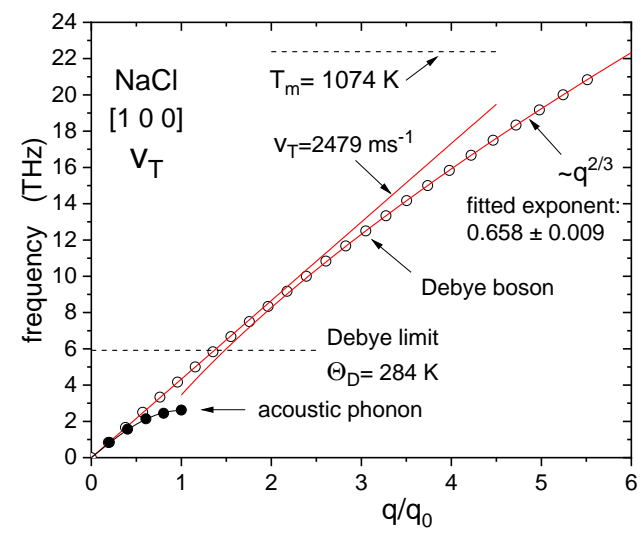

Figure 11. Dispersion of the Debye bosons (circles) [12] and of the acoustic phonons (filled circles) [38], both for transverse polarization measured at $T=80 \mathrm{~K}$ along the edge of the cubic unit cell of $\mathrm{NaCl}$. High-energy data for the Debye bosons are excellently described, up to an energy of $k_{B} \cdot T_{m}$, by the rational exponent of $x=2 / 3$. Note that at the Debye cut-off energy of $k_{B} \cdot \Theta_{D}$ with $\Theta_{D}=284 \mathrm{~K}(\sim 5.9$ $\mathrm{THz}$ ) the dispersion is continuous. The indicated sound velocity is for $T=80 \mathrm{~K}$, as calculated from the elastic constant $c_{44}(T)$ [12].

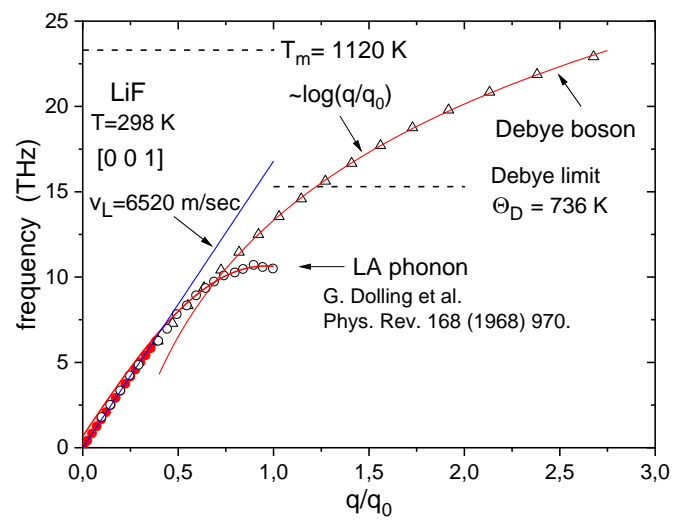

Figure 12. Dispersion of the Debye bosons with longitudinal polarization (open triangles) along cube edge of LiF calculated from the temperature dependence of the sound velocity (elastic constant $\left.c_{11}\right)[6,12,13]$. Fit of the data by a log-function of wave vector means $x=0$. At the Debye cut-off energy at $\sim 15.3 \mathrm{THz}$ the dispersion is continuous. The dispersion of the LA-phonons, measured at T=298 K is given by circles [30]. The fitted sine-function includes a phase shift. The initial slope of the LAphonons agrees with the indicated sound velocity at $T=298 \mathrm{~K}$ [6]. The melting temperature of LiF $\left(T_{m}=1120 \mathrm{~K}\right)$ corresponds to $\sim 23.3 \mathrm{THz}$.

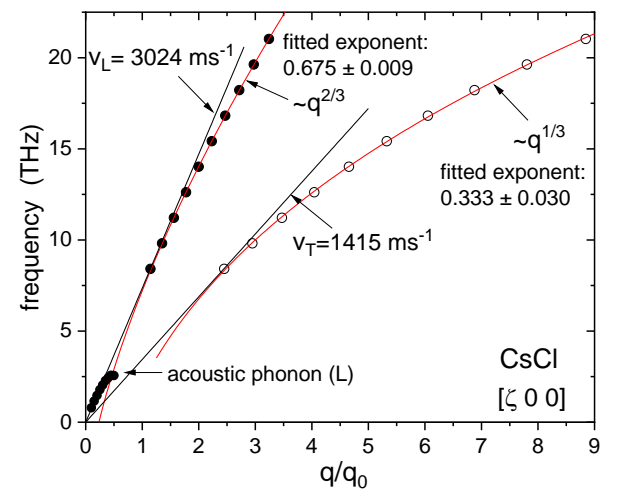

Figure 13. Dispersion of the Debye bosons with longitudinal polarization (filled circles) and with transverse polarization (circles) calculated from the temperature dependence of the sound velocities (elastic constants) for $\mathrm{CsCl}$ [12]. The transverse data confirm existence of the exponent $x=1 / 3$. The dispersion of the acoustic phonons with longitudinal polarization, measured at $T=78 \mathrm{~K}$, is given by solid points [32]. 


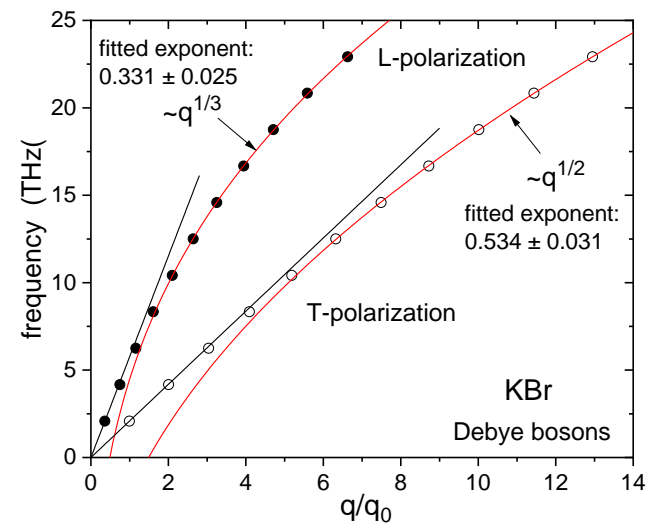

Figure 14. Dispersion of the Debye bosons with longitudinal polarization (dots) and with transverse polarization (circles) calculated from the temperature dependence of the sound velocities [12]. These data for $\mathrm{KBr}$ confirm the exponent values of $x=1 / 3$ and $x=1 / 2$. The initially linear dispersions (straight lines) conform to the sound velocities for $T \rightarrow 0$.

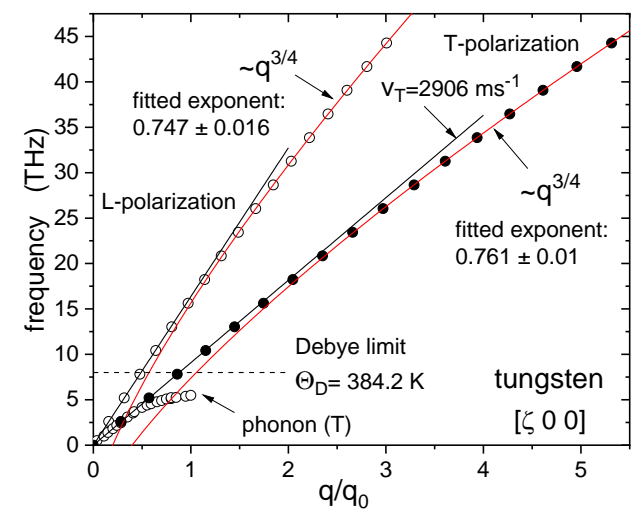

Figure 15. Dispersion of the Debye bosons with longitudinal polarization (circles) and with transverse polarization (filled circles) of tungsten calculated from the temperature dependence of the sound velocities (elastic constants) [12,13]. For both polarizations the exponent is $x=3 / 4$. The dispersion of the phonons with transverse polarization measured at ambient temperature is given by circles [34]. The initial slope of the phonon dispersion, measured at room temperature, is not visibly different from the sound velocity for $T \rightarrow 0$ (straight solid line).

The $q / q_{0}=-A+B \cdot q^{x}$ power functions fitted to the dispersion relations of the Debye bosons (Figures 815) are essentially in the energy (temperature) range between $\sim \mathrm{k}_{\mathrm{B}} \cdot \Theta_{\mathrm{D}}$ and $\sim \mathrm{k}_{\mathrm{B}} \cdot \mathrm{T}_{\mathrm{m}}$. In this temperature range the heat capacity is exclusively due to the MPbosons. We therefore have to investigate whether the curvature in the dispersion of the Debye bosons could alternatively be caused by interactions with the MP-boson. One argument against this is that direct interactions between two boson types are generally unlikely. Debye bosons and photons do also not interact. If there is an interaction between Debye bosons and MP-bosons it can be assumed that this interaction involves the elastic degrees of freedom. It is therefore useful to have a look on the behavior of the lattice expansion data in the temperature range between $\Theta_{D}$ and $T_{m}$. In Figure 16 the temperature dependence of the coefficient of the linear thermal expansion of cubic aluminum, $\alpha(T)$, is plotted as a function of temperature (large red dots) [39]. Additionally, heat capacity data of aluminum up to melting temperature are shown (small black dots) $[14,15]$. In the range below the D-P limit the heat capacity data of $\mathrm{Al}$ have been matched to the $\alpha(\mathrm{T})$ data. As can be seen, for heat capacity values of lower than the D-P limit there is a perfect proportionality between both quantities. This is the so called Grüneisen relation. However, approximately at the Debye temperature a crossover occurs for both quantities as a function of temperature. Moreover, for temperatures of larger than the crossover temperature the two quantities are no longer proportional to each other. While the heat capacity data can excellently be described by a power function of type $\sim\left(\mathrm{T}_{\mathrm{m}}-\mathrm{T}\right)^{\varepsilon}$, the $\alpha(\mathrm{T})$ data follow a power function of absolute temperature of the type $\alpha(T)=c+d \cdot T^{\delta}$. The $\sim\left(T_{m}-T\right)^{\varepsilon}$ function is a typical critical power function of a boson field with $\mathrm{T}_{\mathrm{m}}$ (melting temperature) as critical temperature. The large temperature range and the rational exponent of $\varepsilon=2 / 3$ prove that in the temperature range of the $\sim\left(\mathrm{T}_{\mathrm{m}}-\mathrm{T}\right)^{\varepsilon}$ function the MP-bosons are the relevant excitations, and define the universality class of the heat capacity exclusively. On the other hand, the power function of absolute temperature for $\alpha(\mathrm{T})$ indicates that the associated critical temperature is $\mathrm{T}=0$. This agrees with the Debye boson field that is not ordered at any finite temperature. The critical temperature of the Debye boson field therefore is $\mathrm{T}=0$. The absolute constant in the $\alpha(\mathrm{T})$ function is typical for a non-asymptotic behavior (see discussion of Figure 2).

As a conclusion, it appears that the only signature of the MP-bosons is their heat capacity. Since $\alpha(\mathrm{T})$ seems to be due to the Debye bosons, it follows that the MP-bosons are not -or much weaker- involved in the thermal lattice expansion than the Debye bosons. In other words, the MP-bosons do nearly not couple 
to the elastic degrees of freedom. This is an unexpected result since the MP-bosons can be considered to warrant the cohesion of the solid for thermal energies of larger than the inter-atomic interaction energies as they can be estimated from the largest phonon energies. On the other hand, virtually no interaction of the MP-bosons with the elastic degrees of freedom may explain the first order character of the melting transitions of all solids $[15,16]$.

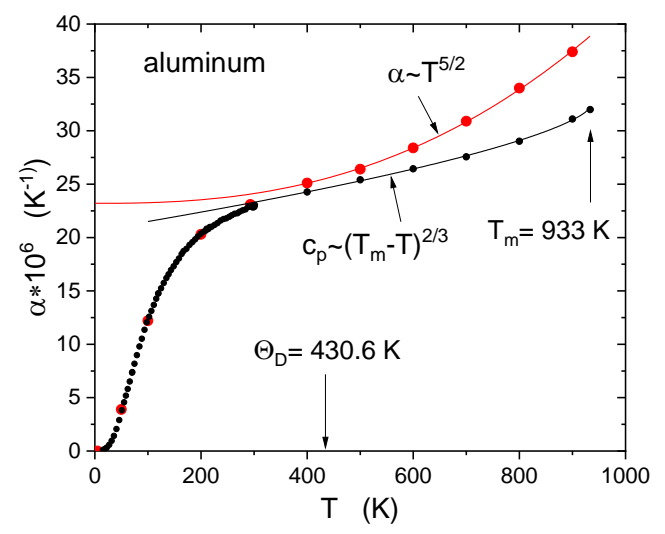

Figure 16. Temperature dependence of the coefficient of the linear thermal expansion, $\alpha(T)$, of cubic aluminum (large red dots) [39]. In the temperature range below the D-P limit $\left(T<\Theta_{D}\right)$, the heat capacity data of aluminum (small black dots) have been matched to the $\alpha(T)$ data $[8,15]$. The two quantities are proportional to each other (Grüneisen relation) below the D-P limit only. Near to the Debye temperature, viz. to the D-P limit, both quantities exhibit a crossover as a function of temperature and are no longer proportional to each other (for details see text).

Since the dispersion of the Debye bosons follows a power function of type $E(q)=-A+B \cdot q^{x}$ with rational exponent $\mathrm{x}$, it can be expected that the coefficient of the linear thermal expansion follows a similar power function as a function of temperature, with also a rational exponent. This conclusion assumes that the Debye bosons are responsible also for the temperature dependence of the thermal lattice expansion. In fact, for temperatures that correspond to the fit range of the function $E(q)=-A+B \cdot q^{x}$, the $\alpha(\mathrm{T})$ data can excellently be described up to melting temperature by a power function of type $\alpha(\mathrm{T})=\mathrm{c}+\mathrm{d} \cdot \mathrm{T}^{\delta}$ with a rational exponent $\delta$ (Figure 17). In particular, for both functions, $E(q)$ and $\alpha(T)$, the applicability starts at the same thermal energy of $\sim \mathrm{k}_{\mathrm{B}} \cdot \Theta_{\mathrm{D}}$. Note that the dispersion energies of the Debye bosons according to $\mathrm{E}(\mathrm{q})=-\mathrm{A}+\mathrm{B} \cdot \mathrm{q}^{\mathrm{x}}$ entail no heat capacity since the dispersion relation of the Debye bosons is thermally not populated in the fit range of the $E(q)=-A+B \cdot q^{x}$ function. Nevertheless, excitation of sound waves in this range of thermal energies (temperatures) is possible, and proceeds out of thermal equilibrium. The absolute constants in the power functions of both quantities indicate that these functions are not the asymptotic behavior for $\mathrm{q} \rightarrow 0$ viz. $\mathrm{T} \rightarrow 0$. Figure 17 visualizes for a selection of cubic metals that the temperature dependence of $\alpha(\mathrm{T})$ follows, starting at $\sim \Theta_{\mathrm{D}}$, a single power function of temperature with rational exponent $\delta$ up to melting temperature [39]. As the analyses of many metallic elements and non-metallic compounds [40] with cubic symmetry show, there are more values for $\delta$ than only those of Figure 17. For the here investigated materials the fit results for $\delta$ are $\delta=1 / 2$ $(\mathrm{Nb}, \mathrm{Si}, \mathrm{Li}, \mathrm{Ar}, \mathrm{Kr}, \mathrm{Ge}, \mathrm{Li}$ ), $\delta=1$ (CsI, RbI, MgO, $\mathrm{NiO}), \delta=4 / 3(\mathrm{Ag}, \mathrm{RbCl}, \mathrm{CaO}), \delta=5 / 3(\mathrm{~V}, \mathrm{Pd}, \mathrm{Ne})$, $\delta=5 / 2(\mathrm{Al}, \mathrm{W}, \mathrm{Pb}, \mathrm{NaCl}, \mathrm{NaF}), \delta=3(\mathrm{Mo}, \mathrm{Ni}), \delta=4$ ( Ta, $\mathrm{AgCl}, \mathrm{LiF})$. These exponent values can be grouped into two sequences according to $\delta=3 / 3,4 / 3$, $5 / 3$ and $6 / 3$ and according to the sequence $\delta=1 / 2,2 / 2$, $4 / 2,5 / 2,6 / 2$ and $8 / 2$. The typical fit errors for these exponents are \pm 0.05 . As a conclusion, the power functions of absolute temperature for $\alpha(\mathrm{T})$ exclude that the MP-bosons give the dominant contribution to the thermal lattice expansion. Otherwise power functions of the argument $\left(\mathrm{T}_{\mathrm{m}}-\mathrm{T}\right)$ should be observed.

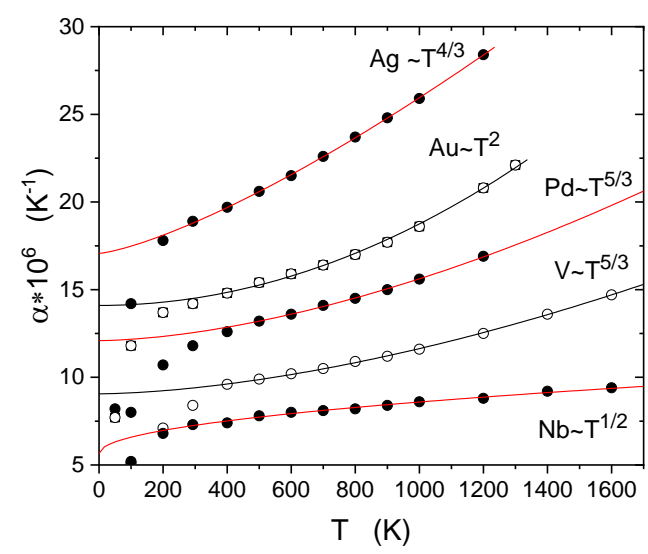

Figure 17. Coefficient of the linear thermal expansion, $\alpha(T)$, of various metallic elements for temperatures of larger than the Debye temperature [39]. Starting from $\Theta_{D}$, these data can be described, up to melting temperature by the power function of absolute temperature of type $\alpha(T)=c+d \cdot T^{\delta}$ with a rational value of the exponent $\delta$ (solid curves). 
It is, however, nearly not possible to correlate the exponents $\delta$ with the other material properties. For instance, the Rare Gas solids $\mathrm{Ne}, \mathrm{Ar}, \mathrm{Kr}$ and $\mathrm{Xe}$ are chemically very similar and all have the cubic fcc structure [20-23]. Nevertheless, the exponents $\delta$ are different. For $\mathrm{Ar}$ and $\mathrm{Kr} \delta=1 / 2$ but for $\mathrm{Ne} \delta=5 / 3$ and for $\mathrm{Xe} \delta=2$.

\section{The Heat Capacity}

As we have seen (Figures 5-7), there are strong indications that the Debye bosons determine the wave-vector dependence of the dispersion of the acoustic phonons. The absolute dispersion energies of the phonons are given by the inter-atomic interactions. In Figures 5-7 a selection of clear examples confirming this peculiarity was given for phonons propagating along $[\zeta 00]$ direction in cubic crystals. If the Debye bosons determine the wavevector dependence of the phonon dispersions it can reasonably be expected that they affect the detailed temperature dependence of the heat capacity of the phonon system as well. It appears that the thermodynamics of solids is generally controlled by bosons and is a phenomenon on the large length scale of the mean free path of these bosons. In insulators with a good thermal conductivity, the mean free path of the Debye bosons can reach a few millimeters in the low-temperature range of the $\mathrm{T}^{3}$ function [6,7]. As a consequence, for samples with a linear dimension of less than the mean free path of the Debye bosons, the low-temperature thermal conductivity is determined by the heat capacity of the Debye boson field and starts with the same universal $\mathrm{T}^{3}$ dependence as the heat capacity $[6,7]$. Since thermal conductivity of insulators is exclusively due to the Debye bosons, a good thermal conductivity requires that the Debye bosons have a heat capacity. This means, the dispersion relation of the Debye bosons must be thermally populated. A good thermal conductivity therefore is limited to temperatures of $\mathrm{T}<\mathrm{T}_{\mathrm{AC}}$ (see Figure 2). Heat transport by phonons is generally negligible [6,7]. For temperatures of $\mathrm{T}>\mathrm{T}_{\mathrm{AC}}$ the phonons come into play and thermal conductivity of the insulators decreases dramatically [6,7]. This results in a rather sharp maximum of the thermal conductivity in the vicinity of $\mathrm{T}_{\mathrm{AC}}$.

As we have explained, between the crossover temperature $\mathrm{T}^{*}$ and that temperature at which the heat capacity has reached the atomistic Dulong-Petit limit (see Figures 3 and 4), the absolute heat capacity values are determined by the phonons. Nevertheless, the dynamics, that is the detailed temperature dependence of the heat capacity is determined by the
Debye bosons. Interestingly, this is for $\mathrm{T}>\mathrm{T}^{*}$ (see Figures 3 and 4) where the dispersion relation of the Debye bosons is thermally no longer populated. For $\mathrm{T}>\mathrm{T}^{*}$, the heat capacity of all insulating solids can well be described by one universal power function of the type $\mathrm{c}=\mathrm{c}_{0}-\mathrm{B} \cdot \mathrm{T}^{-\varepsilon}$. The parameters $\mathrm{c}_{0}$ and $\mathrm{B}$ are material specific and define the absolute heat capacity values. One can expect that the fitted value for the parameter $c_{0}$ agrees with the D-P limit. However, the fit values for $\mathrm{c}_{0}$ are generally larger by $\sim 10 \%$ compared to the D-P limit. The parameter B is given by the inter-atomic interaction strengths, and defines the temperature at which the heat capacity reaches the D-P limit. As a consequence, B scales approximately with the Debye temperature $\Theta_{\mathrm{D}}$. In contrast to the intrinsic $\mathrm{T}^{3}$ function of the heat capacity of the Debye boson field, that holds for all solids at sufficiently low temperatures (see Figure 1), there is not only one universal exponent $\varepsilon$. Depending on the type of interaction between Debye bosons and phonons a number of different exponents $\varepsilon$ can result. In Figures 8-15 strong indications were obtained for the existence of different modes of Debye boson-phonon interaction. As a consequence, different well-defined values for the exponent $\varepsilon$ can be expected. However, as we have explained, the exponents $\mathrm{x}$ and $\varepsilon$ are not simply correlated. Within the experimental accuracy limits, the fit-values for the exponent $\varepsilon$ seem to be rational numbers ranging in the rather narrow interval of $1<\varepsilon<4 / 3$.

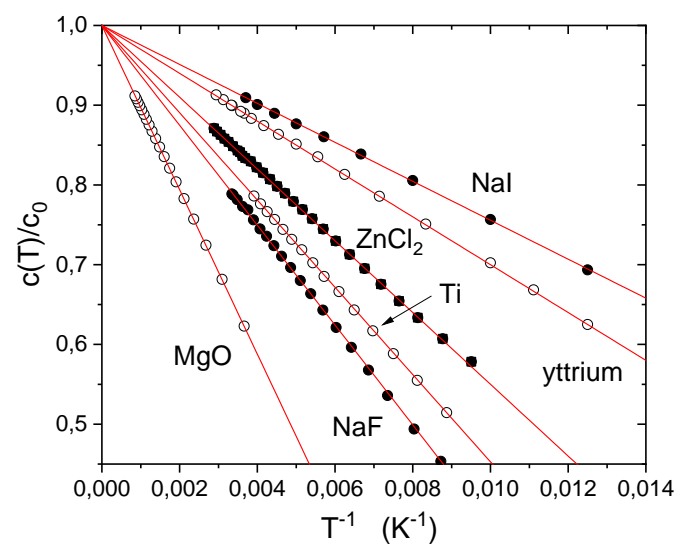

Figure 18. For the selected materials with different chemical compositions and different lattice structures the high-temperature heat capacity is excellently described by the expression $c=c_{0}-B \cdot T^{\varepsilon}$ with $\varepsilon=1$. The parameter $c_{0}$ is the fitted asymptotic heat capacity value for $T \rightarrow \infty$, and is larger than the theoretical Dulong-Petit value. For a properly selected upper and lower limit of the fit range the typical fit error for $\varepsilon$ is \pm 0.015 . 
Evaluation of the exponent $\varepsilon$ from the heat capacity data is more difficult than the evaluation of the exponent $x$ in the dispersion relation of the Debye bosons (Figures 8-15). The main reason for this is the difficulty to correctly select the upper and lower limits of the fit range for the function $\mathrm{c}=\mathrm{c}_{0}-\mathrm{B} \cdot \mathrm{T}^{-\varepsilon}$. These limits are given by the two crossover events at $\mathrm{T}^{*}$ and at that temperature at which the heat capacity approaches the D-P limit, respectively (see Figures 3 and 4). Since crossover events are not absolutely sharp phenomena but are smeared over some finite temperature interval, the fit cannot be conducted up to the crossover temperature but has to be stopped appropriately before. Another ambiguity is that the absolute differences between the different values of $\varepsilon$ are small and often compare with the fit errors for $\varepsilon$. Additionally, the not explicitly specified systematic errors in the experimental heat capacity data can be a problem, in addition to the statistical errors.

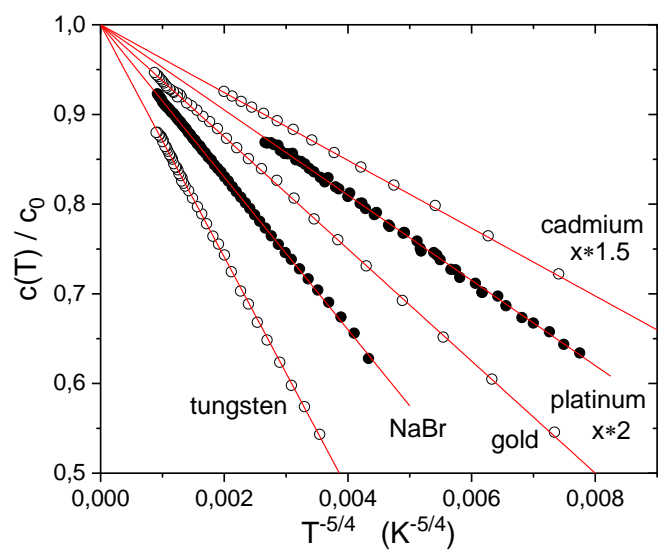

Figure 19. For the selected materials with different chemical compositions and lattice structures the same exponent $\varepsilon=5 / 4$ in the function $c=c_{0}-B \cdot T^{\varepsilon}$ for the high-temperature heat capacity is observed. Note that $A u$ and Pt have fcc structure, W has bcc structure, $\mathrm{NaBr}$ has the cubic $\mathrm{NaCl}$ structure while Cd has hexagonal hcp structure. For better clarity, the abscissa values of the Cd and Pt data are multiplied by the given factors.

One clearly identified exponent value is $\varepsilon=1$. Figure 18 shows a few examples of this exponent $[8,14]$. As can be seen in Figure 18, the heat capacities of chemically different materials with different lattice structures follow the same $\mathrm{c}_{0}-\mathrm{B} \cdot \mathrm{T}^{-1}$ function rather precisely. The exponent $\varepsilon=1$ therefore has to be identified as universal. Note that $\mathrm{MgO}, \mathrm{NaF}$ and $\mathrm{NaI}$ have the $\mathrm{NaCl}$ structure, titanium and yttrium have the hexagonal closed packed structure (hcp) and $\mathrm{ZnCl}_{2}$ has hexagonal structure. In Figure 18 the heat capacity data have been normalized to the fitted asymptotic value for $\mathrm{T} \rightarrow \infty$, $c_{0}$. Note that $c_{0}$ is generally larger than the theoretical D-P value (see Figures 3 and 4). The as normalized heat capacity data are plotted over $\mathrm{T}^{-1}$ in Figure 18. The typical fit error for $\varepsilon$ is \pm 0.015 . It can be seen that the slopes of the fitted $\mathrm{c}=\mathrm{c}_{0}-\mathrm{B} \cdot \mathrm{T}^{-\varepsilon}$ functions (the parameter B) scale approximately with the Debye temperature $\Theta_{\mathrm{D}}$, that are $\Theta_{\mathrm{D}}=942 \mathrm{~K}$ for $\mathrm{MgO}$, $\Theta_{\mathrm{D}}=420 \mathrm{~K}$ for titanium, $\Theta_{\mathrm{D}}=256.4 \mathrm{~K}$ for yttrium and $\Theta_{D}=167.5 \mathrm{~K}$ for $\mathrm{NaI}[4,6]$. We should mention that in the high-temperature heat capacity of the metallic elements the contributions of the Debye bosons $\left(\sim \mathrm{T}^{3}\right)$ and of the EC-bosons $(\sim \mathrm{T})$ are absent. This is because for the temperatures of $\mathrm{T}>\mathrm{T}^{*}$ the dispersion relations of the two boson types are no longer thermally populated. On the other hand, the finite heat capacity of the electronic band states, that is also liner-in- $T$, should be visible. This contribution seems to be negligibly small compared to the lowtemperature heat capacity $c_{\mathrm{el}}=\gamma \cdot \mathrm{T}$ of the EC-bosons $[7,13]$.

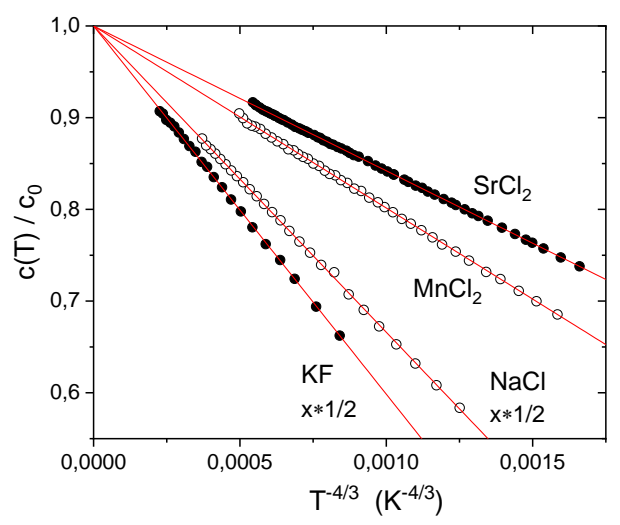

Figure 20. For the selected materials with different chemical compositions and lattice structures, the same exponent of $\varepsilon=4 / 3$ of the function $c=c_{0}-B \cdot T^{\varepsilon}$ is observed in the high-temperature range with $a$ phonon defined heat capacity [8]. Note that KF has the cubic $\mathrm{NaCl}$ structure while $\mathrm{MnCl}_{2}$ has rhombohedral structure but $\mathrm{SrCl}_{2}$ has cubic structure.

Figure 19 displays a few selected examples for the exponent $\varepsilon=5 / 4$. Again, the materials of Figure 19 are chemically different and have different lattice structures. Tungsten has bcc structure, $\mathrm{NaBr}$ has the $\mathrm{NaCl}$ structure, $\mathrm{Au}$ (gold) and $\mathrm{Pt}$ have fcc structure and $\mathrm{Cd}$ has hcp structure. All heat capacity data are normalized to the fitted value for $\mathrm{c}_{0}$ and are plotted 
on $\mathrm{T}^{-5 / 4}$ temperature scale in Figure 19. For the selected materials the typical fit error for $\varepsilon$ is \pm 0.021 .

Figure 20 displays some examples for the exponent $\varepsilon=4 / 3$. Note that $\mathrm{KF}$ has the $\mathrm{NaCl}$ structure, while $\mathrm{MnCl}_{2}$ is hexagonal and $\mathrm{SrCl}_{2}$ has the cubic fluorite structure. The heat capacity data in Figure 20 are again normalized to the fitted value for $\mathrm{c}_{0}$ and are plotted over $\mathrm{T}^{-4 / 3}$. The typical fit error for $\varepsilon$ is \pm 0.026 .

There are, however, more than only the three clearly identified exponent values of $\varepsilon=1,5 / 4$ and $4 / 3$. This can be rationalized since there are at least 6 different modes of interaction between the Debye bosons and the phonons according to the 6 values for the exponent $x(x=0,1 / 4,1 / 3,1 / 2,2 / 3$ and $3 / 4)$ in the dispersion relation of the Debye bosons (Figures 815). The other fit-values for the exponent $\varepsilon$ are all near to $\varepsilon \sim 1.1$ and are more difficult to separate from each other. As an average over many analyzed materials the exponent $\varepsilon=1.153 \pm 0.025$ could reasonably be established. This exponent value appears to be a non-rational number. Since in the same material different exponents $\mathrm{x}$ occur for different polarizations of the Debye bosons, different modes of interaction can be simultaneously active. It is possible that the different types of interaction interfere. In fact, $\varepsilon=1.153 \pm 0.025$ could be interpreted as the average of $\varepsilon=1$ and $\varepsilon=5 / 4$, that is $\varepsilon=1.125$ but also as the average of $\varepsilon=1$ and $\varepsilon=4 / 3$, that is $\varepsilon=1.167$. Interference exponents are known from magnetic systems in which different universality classes coexist [6]. This applies to magnetic materials with two types of magnetic atoms that have integer or half-integer spin.

\section{Discussion}

The conclusions presented in this communication are rather preliminary and need further experimental studies for a final approval. Many statements should be considered as working hypotheses only. However, since the advent of the Renormalization Group (RG) methods it became clear that boson fields are of fundamental importance for the dynamics in solids. This calls for the development of appropriate field theories. It is evident that the prerequisite for this task is that the field quanta are precisely known. Quite generally, future theories of the dynamics of solids have to distinguish properly between the two translational symmetries: the discrete and periodic translational symmetry of the atomic structure and the continuous translational symmetry of the infinite solid. This distinction has to be made for the elastic, the magnetic and the electronic degrees of freedom. The two translational symmetries are the generators of specific particles with characteristic excitation spectra. In the elastic case the two particles are well-known as phonons and sound waves [13]. The latter we have called Debye bosons. Unfortunately, in the first field theory of the heat capacity of solids by P. Debye in 1912 [2], no explicit differentiation between the two translational symmetries and their excitation spectra was made [2]. Consequently, crossover events were ignored. The conceptual deficiencies of Debye's theory could be resolved only seven decades later by the development of the RG methods [3]. As we have shown earlier, the bosons of the continuous magnetic solid are essentially magnetic dipole radiation generated by the processing spins $[6,28]$. We have called them Goldstone bosons. The excitations of the discrete magnetic lattice are the well-known magnons.

The excitations of the continuous translational symmetry (bosons) and of the discrete translational symmetry (phonons, magnons) are, however, not independent of each other, but interact significantly. This is a considerable complication for the development of realistic field theories of solid-state physics. Here we have focused on the interaction between the Debye bosons and the phonons that is phenomenologically very similar to the interaction between Goldstone bosons and magnons in the magnetic materials [41]. Due to this interaction the dispersion relations of the acoustic phonons (magnons) deviate characteristically from the conventional, purely atomistic descriptions. In other words, the dispersion relations of phonons (magnons) cannot be understood by neglecting the interactions with the Debye bosons (Goldstone bosons). This has the severe consequence that the interaction parameters between neighboring atoms (spins) cannot be evaluated reliably from the experimental phonon (magnon) dispersion relations. It appears that only the absolute energies of the phonons (magnons) are determined by the interatomic interactions but that the wave-vector dependence of their dispersion relations is determined by the bosons. As we have shown, the dispersion relations of the acoustic phonons (magnons) along the $[\zeta, 0,0]$-direction in cubic solids are essentially as for the linear atomic chain (spin chain), i.e. one-dimensional [27,41]. This is unusual for a three-dimensional solid and has been explained by a dominating stimulated emission in the generation process of both boson types. Stimulated emission is essential for the perfect one-dimensional ordered Goldstone boson fields and the onedimensional magnon dispersions within each magnetic domain. Since the Debye boson field is not 
ordered (its critical temperature is $\mathrm{T}=0$ ), the phonon dispersion as for the linear atomic chain has to be considered as of short-range only. Note that inelastic neutron scattering provides no evidence of a coherent long-range order. The spontaneous generation of the Debye bosons by individual atoms therefore seems to be dominated by stimulated emission as well. This generation process is, however, completely unexplored theoretically. The observed universality in the temperature dependence of the heat capacity of the atomistic phonons or magnons can be understood as a direct consequence of their boson-determined dispersion relations [41].

One origin of the phenomenon of broken symmetry at phase transitions appears to be that the generation process of most boson types seems to be by stimulated emission. In the magnetic case this can be rationalized, since the field bosons are essentially magnetic dipole radiation [28]. However, there exists no theory yet for the generation of magnetic dipole radiation by the precessing spins, but it is known that this process is dominated by stimulated emission. As a consequence, the basic ordered Goldstone boson field is perfectly one-dimensional, as it is realized in each magnetic domain. A two- or three-dimensional global boson field results by some (dynamic) coupling of the boson fields (domains) along two- or three in-equivalent crystal axes. The necessary coupling between the domains defines the dynamic dimensionality of the resulting global boson field.

Near critical temperatures, the dynamics seems generally to be determined by boson fields as reveals by the observation of universality. One obvious example for this is the critical temperature of $\mathrm{T}=0$ of the Debye boson field. On approaching $\mathrm{T} \rightarrow 0$ the heat capacity of the non-magnetic insulators is exclusively due to the Debye boson field; the assumed heat capacity contributions of the atomistic phonons are completely suppressed, i.e. nonrelevant. In other words, all thermal energy is in the boson field. In the same way, the atomistic exchange interactions become non-relevant on approaching the finite magnetic ordering temperature from the paramagnetic side $[3,6,27,41]$. This is the basis for the universality of the critical magnetic behavior. Non-relevance of the atomistic exchange interactions reveals clearly from the fact that the critical behavior is identical for ferromagnets and for antiferromagnets.

We can assume that the melting process of the solids is also controlled by a boson field [16]. We have called the not yet specified bosons at the melting point MP-bosons. This boson field seems to be responsible for the coherent long-range atomic order in the solid phase and guarantees the cohesion of the solid for thermal energies of larger than the inter-atomic interaction energies, that correspond approximately to $\mathrm{E}>\mathrm{k}_{\mathrm{B}} \cdot \Theta_{\mathrm{D}}$. The MP-bosons therefore have a binding function. A long-range and coherent atomic or magnetic order seems to be generally due to a coherent boson field. As can be concluded from the rational critical exponents and the finite width of the critical range, the vapor-liquid transition seems to be driven also by a boson field $[18,19]$. The associated bosons are, however, completely unexplored as yet.

\section{Acknowledgments}

The work of this publication is not the sole merit of the author. Without the fantastic and meticulous data collections of R.F.S. Hearmon, of Y.S. Touloukian and co-authors and of I. Barin a systematic analysis of so many elastic, spectroscopic and calorimetric data on a huge number of materials would not have been possible. These data were gained over the last seven decades by numerous excellent and ingenious experimentalists. It is very satisfying that all these carefully measured and collected data are not only available for practical use, but contain unrecognized and indispensable basic information for the achievement of a more profound and satisfying understanding of the dynamics of solids. I extend my thanks to all of the excellent experimentalists that have produced the accurate data that were of fundamental importance for the new findings presented here.

\section{References}

[1] M. Born, K. Huang, Dynamical Theory of Crystal Lattices, Clarendon Press, Oxford, 1956.

[2] P. Debye, Zur Theorie der spezifischen Wärme, Ann. Physik, 39, 789-839, 1912.

[3] K.G. Wilson, J. Kogut, The renormalization group and the $\varepsilon$ expansion, Physics Reports, 12C, 75-200, 1974.

[4] G.A. Alers, Use of Sound Velocity Measurements in Determining the Debye Temperature of Solids, in: Physical Acoustics, ed. by W.P. Mason, vol III B, Academic Press, New-York,1-42, 1965.

[5] W.S. Corak, M.P. Garfunkel, C.B. Satterthwaite, A. Wexler, Atomic Heats of Copper, Silver and Gold from $1{ }^{0} \mathrm{~K}$ to $5{ }^{0} \mathrm{~K}$, Phys. Rev. 98, 1699-1708, 1955. 
[6] U. Köbler, A. Hoser, Experimental Studies of Boson Fields in Solids, World Scientific, Singapore, 2018.

[7] U. Köbler, On the Thermal Conductivity of Metals and of Insulators, JIoT, 20, 210-218, 2017.

[8] Y.S. Touloukian, E.H. Buyco, Thermophysical Properties of Matter, vol 5: Specific Heat of Nonmetallic Solids, IFI/Plenum, New-York, 1970.

[9] T.H.K. Barron, W.T. Berg, J.A. Morrison, On the heat capacity of crystalline magnesium oxide, Proc. Roy. Soc. (London) A250, 70$83,1959$.

[10] J.C. Ho, D.P. Dandekar, Low-temperature heat capacities of $\mathrm{RbCl}, \mathrm{RbBr}$ and $\mathrm{CsCl}$, Phys. Rev. B 30, 2117-2119, 1984.

[11] W.T. Berg, J.A. Morrison, The heat capacity of potassium chloride, potassium bromide potassium iodide and sodium iodide, Proc. Roy. Soc. (London) A242, 467-477, 1957.

[12] R.F.S. Hearmon, The elastic constants of crystals and other anisotropic materials, in: Landolt-Börnstein, ed. by K.-H. Hellwege, vol. III/11, 1-286, Springer, Berlin 1979.

[13] U. Köbler, On the Distinction between Debye Bosons and Acoustic Phonons, IJoT, 18, 277284, 2015.

[14] Y.S. Touloukian, E.H. Buyko, Thermophysical Properties of Matter, vol. 4: Specific Heat of Metallic Elements and Alloys, IFI/Plenum, New-York, 1970.

[15] I. Barin, Thermochemical Data of Pure Substances, $3^{\text {th }}$ edition, VCH, Weinheim, 1995.

[16] U. Köbler, V.Yu. Bodryakov, On the Melting Process of Solids, IJoT, 18, 200-204, 2015.

[17] M.E. Fisher, A.N. Berker, Scaling for first-order phase transitions in thermodynamic and finite systems, Phys. Rev. B 26, 2507-2513, 1982.

[18] P. Heller, Experimenal investigations of critical phenomena, Rep. Prog. Physics 30, 731-826, 1967.

[19] R.K. Pathria, Statistical Mechanics, $2^{\text {th }}$ edition, Butterworth-Heinemann, Oxford 1996.

[20] Y. Fujii, N.A. Lurie, R. Pynn, G. Shirane, Inelastic neutron scattering from solid ${ }^{36} \mathrm{Ar}$, Phys. Rev. B 10, 3647-3659, 1974.

[21] Y. Endoh, G. Shirane, J. Skalyo, Jr., Lattice dynamics of solid neon at 6.5 and $23.7 \mathrm{~K}$, Phys. Rev. B 11 (1975) 1681-1688, 1975.

[22] J. Skalyo, Jr., Y. Endoh, G. Shirane, Inelastic neutron scattering from solid krypton at $10{ }^{\circ} \mathrm{K}$, Phys. Rev. B 9 1797-1803, 1974.
[23] N.A. Lurie, G. Shirane, J. Skalyo, Jr., Phonon dispersion relation in xenon at $10 \mathrm{~K}$, Phys. Rev. B 9, 5300-5306, 1974.

[24] C. Stassis, G. Kline, W. Kamitakahara, S.K. Sinha, Lattice dynamics of fcc ${ }^{4} \mathrm{He}$, Phys. Rev. B 17 1130-1135, 1978.

[25] H. Bilz, W. Kress: Phonon Dispersion Relations in Insulators, Springer, Berlin, 1979.

[26] R.W.G. Wyckoff, Crystal Structures, vol.1, R.E. Krieger Publ. Comp. Malabar, Florida 1982.

[27] A. Hoser, U. Köbler, Functional crossover in the dispersion relations of magnons and phonons, J. Phys: Conf. Series 746, 012062-8, 2016.

[28] A. Hoser, U. Köbler, Boson Fields in Ordered Magnets, Acta Phys. Pol. A 127, 350-352, 2015.

[29] E.C. Svensson, B.N. Brockhouse, J.M. Rowe, Crystal Dynamics of Copper, Phys. Rev. 155, 619-632, 1967.

[30] G. Dolling, H.G. Smith, R.M. Nicklow, P.R. Vijayaraghavan, M.K. Wilkinson, Lattice Dynamics of Lithium Fluoride, Phys. Rev. 168, 970-979, 1968.

[31] L. Pintschovius, W. Reichardt, B. Scheerer, Lattice dynamics of TiC, J. Phys. C: Solid State Phys. 11, 1557-1562, 1978.

[32] A.A.Z. Ahmad, H.G. Smith, N. Wakabayashi, M.K. Wilkinson, Lattice Dynamics of Cesium Chloride, Phys. Rev. B 6, 3956-3961, 1972.

[33] M. Steiner, B. Dorner, J. Villain, Inelastic neutron investigation of the anisotropy of the spin wave linewidth in the one-dimensional easy-plane ferromagnet $\mathrm{CsNiF}_{3}$, J. Phys. C: Solid State Phys. 8, 165-175, 1975.

[34] A. Larose, B.N. Brockhouse, Lattice vibrations in tungsten at $22{ }^{\circ} \mathrm{C}$ studied by neutron scattering, Can. J. Phys. 54 1819-1823, 1976.

[35] N. Vagelatos, D. Wehe, J.S. King, Phonon dispersion and phonon density of states for $\mathrm{ZnS}$ an ZnTe, J. Chem. Phys. 60, 3613-3618, 1974.

[36] M.M. Elcombe, The lattice dynamics of strontium fluoride, J. Phys. C: Solid State Phys. 5, 2702-2710, 1972.

[37] Y.L. Yarnell, J.L. Warren, S.H. Koenig, Experimental Dispersion Curves for Phonons in Aluminum, in: Lattice Dynamics, Proc. Int. Conf. Copenhagen, ed. by R.F. Wallis, 57-61, Pergamon Press, 1963.

[38] G. Raunio, S. Rolandson, Lattice Dynamics of $\mathrm{NaCl}, \mathrm{KCl}, \mathrm{RbCl}$ and RbF, Phys. Rev. B 2, 2098-2103, 1970.

[39] Y.S. Touloukian, R.K. Kirby, R.E. Taylor, P.D. Desai, Thermophysical Properties of Matter, vol.12, Thermal Expansion of Metallic 
Elements and Alloys, IFI/Plenum, New YorkWashington, 1975.

[40] Y.S. Touloukian, R.K. Kirby, R.E. Taylor, T.Y.R. Lee, Thermophysical Properties of Matter, vol.13, Thermal Expansion of
Nonmetallic Solids, IFI/Plenum, New YorkWashington, 1977.

[41] U. Köbler, Bosonic and magnonic magnon dispersions, J. Magn. Magn. Mater. 502, 166533, 1-18, 2020. 